\title{
PROBING AND CONTROLLING PHOTOTHERMAL HEAT GENERATION IN \\ PLASMONIC NANOSTRUCTURES
}

\author{
By \\ Zachary J. Coppens \\ Thesis \\ Submitted to the Faculty of the \\ Graduate School of Vanderbilt University \\ In partial fulfillment of the requirements \\ for the degree of
}

MASTER OF SCIENCE

in

Mechanical Engineering

December, 2012

Nashville, Tennessee

Approved:

Professor Deyu Li

Professor Jason G. Valentine

Professor D. Greg Walker 


\section{DEDICATION}

To my family, whose loving support throughout the years will forever be appreciated

and

To my amazing wife Katie, for her constant love, patience, and encouragement

throughout this process 


\section{ACKNOWLEDGEMENTS}

This thesis would not have been possible without the tremendous guidance and support from my two advisors Jason Valentine and Greg Walker. Their enthusiasm for knowledge and demand for perfection made this project a valuable and enriching learning experience. I will always consider them mentors and friends. Also, this thesis would not have been possible without the generous financial support from Sandia National Laboratories. 


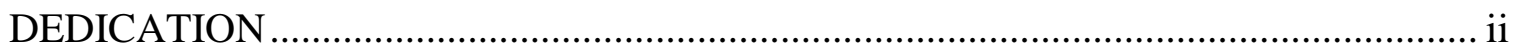

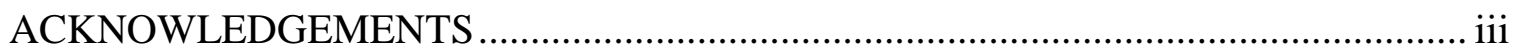

LIST OF FIGURES ………………………………........................................ vi

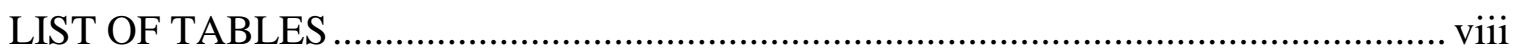

Chapter

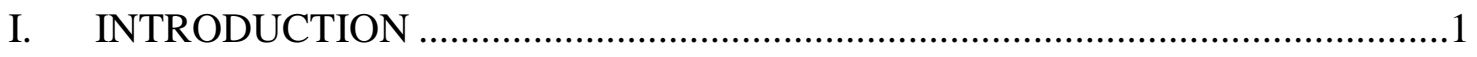

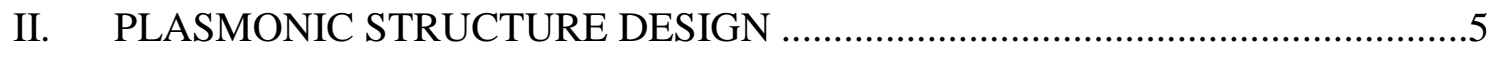

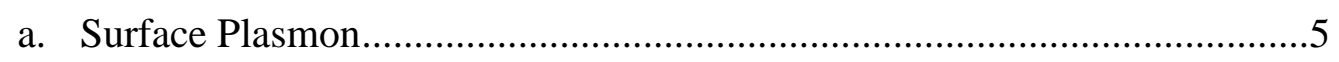

b. Thermoplasmonics Background and Previous Work ...................................

c. Proposed Thermoplasmonic Design Rationale ............................................11

d. Enhanced Thermoplasmonic Antenna Design ...........................................12

e. Nanoparticle Superstructure....................................................................17

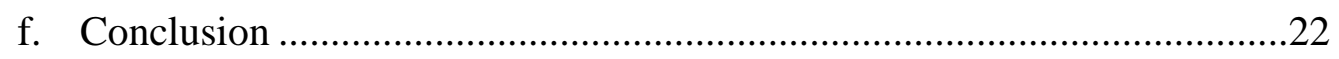

III. MICRO/NANOSCALE TEMPERATURE MEASUREMENTS .........................23

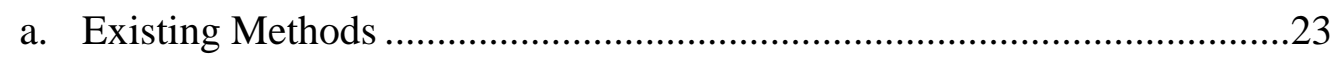

b. Proposed Micro/nanoscale Temperature Measurement Technique ............25

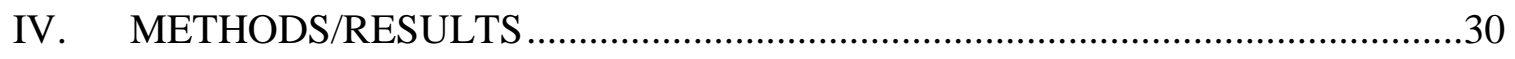

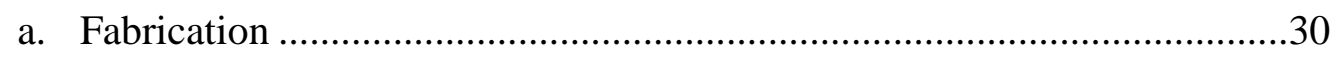

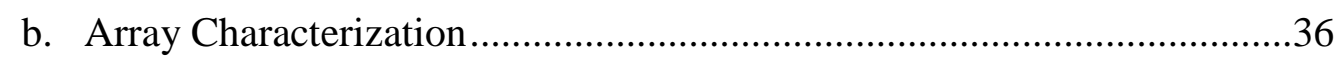




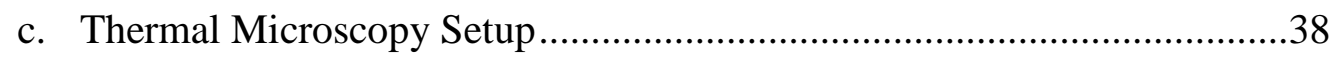

d. Calibration and Array Temperature Measurements...............................43

V. THERMAL MODELING ...................................................................... 47

a. Optically Heated Nanoantenna Arrays .............................................47

b. Nanoparticle Superstructure.........................................................51

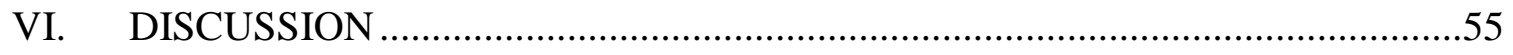

a. TGP Thermal Microscopy Technique ................................................55

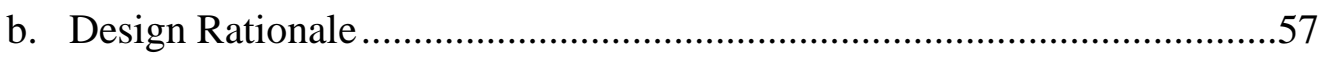

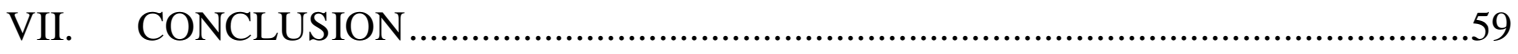

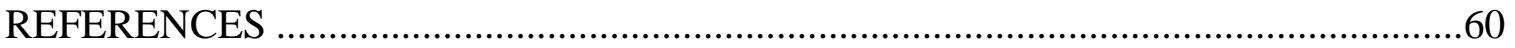




\section{LIST OF FIGURES}

1. Depiction of a surface plasmon wave propagating along the interface of a metal and dielectric material

2. Optically excited localized surface plasmon..................................................

3. Examples of feed gap antenna geometries ................................................

4. Collective thermal effects of nanoparticle arrangements....................................

5. Configuration of plasmonic nanoparticle superstructure ..................................10

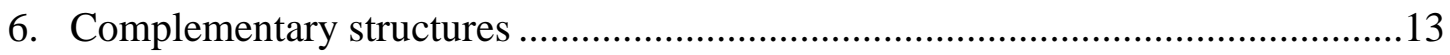

7. Evolution in geometry from dipole to diabolo antenna ....................................15

8. Vectorial representation of current flow in diabolo nanoantenna........................17

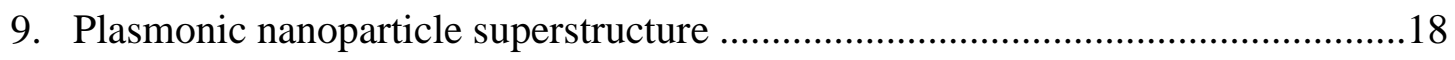

10. Surface plasmon dispersion curve plotted with the light line ............................19

11. Magnetic field profile of superstructure ......................................................21

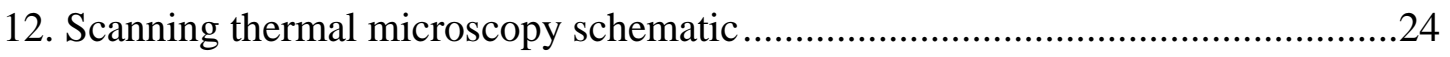

13. Concept of TGP thermal microscopy technique ................................................26

14. Decay plots for varying phosphor temperatures ............................................28

15. Calibration curve of lanthanum oxysulfide taken at $514 \mathrm{~nm}$ emission peak ........29

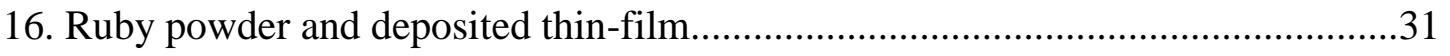

17. Emission spectra of annealed and un-annealed ruby phosphor films ...................32

18. AFM image of deposited ruby film on sapphire substrate ..................................32

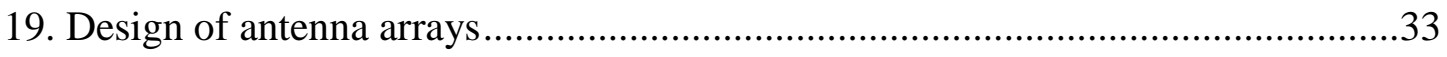

20. Cross-sectional depiction of sample after EBL exposure and $\mathrm{Cr} / \mathrm{Au}$ deposition...35 
21. SEM images of the diabolo antenna array and dipole antenna array.

22. Plot of the simulated and experimental transmission data .38

23. Thin-film TGP thermal microscopy schematic

24. Picture of thermal microscopy setup.

25. Picture of microscope export

26. Log plot of an averaged decay reading from the ruby phosphor .42

27. Schematic of calibration setup .

28. Calibration curve of $100 \mathrm{~nm}$ ruby TGP

29. Temperature measurements of optically excited dipole and diabolo arrays with non-resonant and resonant polarizations.

30. Depiction of the conduction model for a heated dipole antenna array .48

31. Experimental temperature measurements and results from the analytical conduction model.

32. Depiction of conduction model for the diabolo antenna.

33. Plasmonic superstructure made up of a diabolo antenna surrounded by 3 circular gratings

34. Two photon excitation of ruby phosphor layer .56 


\section{LIST OF TABLES}

1. Effects of Metal on TGP Decay Time Measurements ......................................43 


\section{CHAPTER I}

\section{INTRODUCTION}

\section{Motivation}

The ability of surface plasmons to control electromagnetic energy in deep subwavelength volumes has attracted much attention in nanoscale science. In localized surface plasmons, electromagnetic fields at optical and near-infrared frequencies are used to excite resonant charge oscillations in metallic nanostructures. These charge oscillations generate highly confined and enhanced electromagnetic fields, an effect that is useful for applications involving sensing, energy conversion, imaging, and optical modulation ${ }^{1}$. However, the major drawback for applications using surface plasmons is that resonant oscillations in metallic structures generate parasitic absorption losses due to Joule heating in the metal. Much of the work involved in the field of plasmonics has been devoted to minimizing this heating loss.

Recently, however, research efforts have shifted toward engineering the loss in plasmonic structures to generate useful heating. This emerging branch of plasmonics, deemed thermoplasmonics, focuses on designing efficient, optically-induced nanoscale heat sources ${ }^{2-5}$. The ability to generate point-like heat sources is not only useful for probing the fundamentals of nanoscale heat transfer, but has potential for several important applications such as medical therapy ${ }^{6-9}$, heat-assisted magnetic recording ${ }^{10,11}$, 
chemical catalysis ${ }^{12-15}$, phononic circuitry ${ }^{16,17}$, thermal lithography ${ }^{18}$, and thermophotovolatics ${ }^{19,20}$. This area has only recently attracted interest from researchers, yet initial results are promising.

In the field of thermoplasmonics, there are two challenges at the forefront: particle design and micro/nanoscale temperature measurement. Current thermoplasmonic design studies have focused on understanding the thermal response of simple particles such as spheres, disks, core-shell structures, and rods ${ }^{2-5,21}$. These studies have detailed the mechanisms for thermoplasmonic heating and have offered several simple design criteria to increase heat generation. However, in order to fully exploit the potential of thermoplasmonics, a design rationale to explain how more complex geometries can be used to control the magnitude of heat generation in these structures is needed. Furthermore, because measuring the temperature of micro/nanoscale devices cannot follow conventional methodologies, other techniques are needed ${ }^{22}$. Previous studies exploring micro/nanoscale temperature measurements have proposed several promising methods, yet each has its drawbacks. Currently, no micro/nanoscale temperature measurement technique has seen widespread adoption.

In this thesis, a new thermoplasmonic design rationale and micro/nanoscale temperature measurement technique are proposed. The proposed design rationale explains how complex geometries, such as the diabolo nanoantenna, can increase heat generation through enhanced current densities and magnetic fields. It is shown that the key to this rationale is enhancing the magnetic field around nanostructures, which when properly implemented can produce large heat source densities due to non-linear scaling relations. The proposed temperature measurement technique uses the temperature 
dependent decay time of thin-film thermographic phosphors (TGP) for robust thermal microscopy measurements. The fundamental aspects of this technique allow it to overcome the shortcomings of current methods and make it a strong candidate for widespread adoption into micro/nanoscale science.

\section{Organization}

The following chapters of this thesis are broken up into six parts: plasmonic structure design, micro/nanoscale temperature measurements, methods/results, thermal modeling, discussion, and conclusions. In the plasmonic structure design section, background information on plasmonics and previous thermoplasmonic findings are

presented. This section is also used to prove, through mathematical derivation and simulated results, the proposed thermoplasmonic design rationale. The micro/nanoscale temperature measurement section highlights several current temperature measurement techniques including both near-field and far-field methods. The background information for the prosed TGP thermal microscopy method is also presented in this section.

The methods/results section describes the experiments performed to measure the temperature of optically heated, rationally designed nanostructures using the TGP thermal microscopy technique. These measurements are used to validate the derivation and simulations presented in the plasmonic structure design section. The thermal modeling part of this thesis describes two modeling methods. One method is used to validate the experimental results from the TGP temperature measurements, and the other is used to map the temperature distribution around a 
super enhanced thermoplasmonic design. Finally, the discussion section examines aspects of the thermoplasmonic design rationale and includes a discussion of the advantages and limitations of the proposed TGP thermal microscopy technique. 


\section{CHAPTER II}

\section{PLASMONIC STRUCTURE DESIGN}

This chapter will focus on the theory and design of plasmonic and thermoplasmonic structures. First, background information on surface plasmons and plasmonic heating processes is presented. Then, an overview of key findings in previous thermoplasmonic design studies is described. Using both of these areas as background, a new thermoplasmonic design rationale is proposed through mathematical derivation. Finally, the validity of this rationale is shown with finite-difference time-domain (FDTD) simulations of thermoplasmonic structures.

\section{Surface Plasmon}

All plasmonic and thermoplasmonic applications take advantage of a surface plasmon, which is simply the oscillation of an electron cloud at the interface of a metal and dielectric material (Figure 1). Beyond the interface, plasmon fields exponentially decay into both the metal and dielectric, thus providing strong field confinement at the surface $^{1,23}$. Excitation of surface plasmons can result in either propagating modes along the metal/dielectric interface (plasmon polariton) or localized fields in a single metallic particle (localized surface plasmon $)^{23}$. In this thesis, the field confinement of localized surface plasmons is used to generate heat in metal nanoparticles. 


\section{Dielectric}

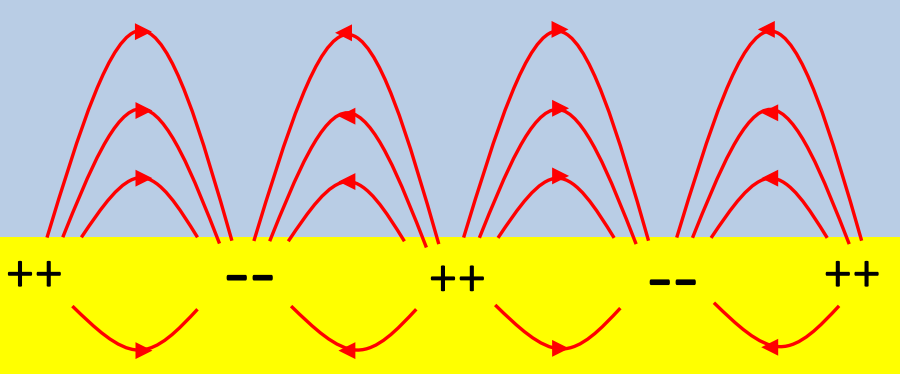

\section{Metal}

Figure 1. Depiction of a surface plasmon wave propagating along the interface of a metal and dielectric material.

Figure 2 shows an optically excited, localized surface plasmon on a metal nanoparticle. The dielectric medium surrounding the metal particle acts as a cavity at the interface of the two materials. This cavity can cause the electromagnetically induced, dipolar oscillations of the metal nanoparticle's electron cloud to become resonant under the condition that the oscillations constructively interfere at the dipolar edges ${ }^{24}$. Outside the quasi-static approximation, this occurs when the dimension of the nanoparticle is half the wavelength of the surface plasmon. In this regard, increasing the dimension of the particle will red-shift the resonance and decreasing the dimension of the particle will blue-shift the resonance. This allows for the plasmonic particle resonance to be tuned for specific applications. The resonant property of localized surface plasmons is important for thermoplasmonics because maximum heat generation occurs at the surface plasmon resonant frequency. 


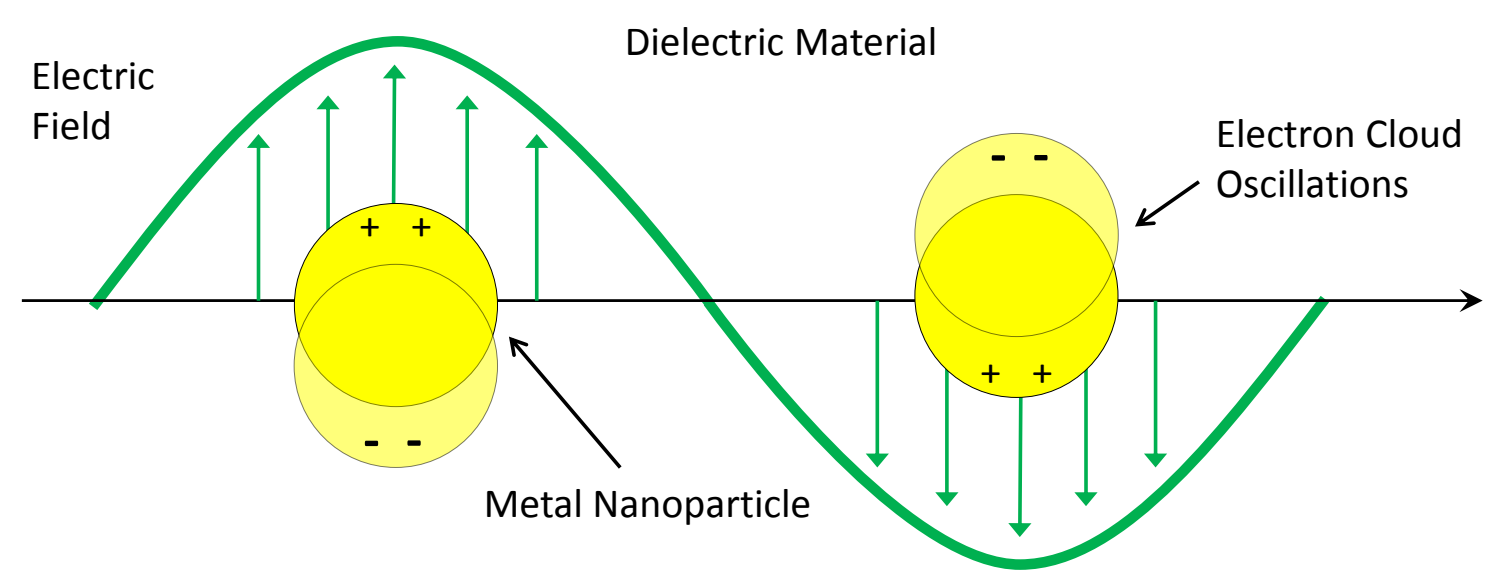

Figure 2. Optically excited, localized surface plasmon.

\section{Thermoplasmonics Background and Previous Work}

Optically induced heating in a plasmonic particle occurs in a three-step process ${ }^{25}$.

First the free electrons of the metal particle undergo rapid oscillations at the resonant frequency and their electronic temperature becomes very high. Next, the energetic electrons begin to scatter with the lattice of the particle, which converts the energy into phonons, or heat carriers. Finally, the phonons generated in the particle propagate to the adjacent dielectric material, thus transferring heat to the surroundings. These three processes together are completed at a time scale on the order of nanoseconds ${ }^{26}$.

Previous work on thermoplasmonic particle heating has focused on understanding the thermal response of simple structures and arrangements, and has provided a basis for the current thermoplasmonic design approaches. One of these early studies showed that thinner structures generate more heat than thicker structures ${ }^{3}$. This is because, when comparing constant volumes, thinner structures allow the plasmon to penetrate further into the core of the structures, thus allowing for more of the lossy metallic material to be 
involved in the heating process. Another study looked at using different optical antenna geometries to enhance electromagnetic fields and generate more heat ${ }^{3,4}$. However, good electromagnetic antennas, such as those with feed gaps, showed a decrease in heat generation. This is because the feed gap geometry (Figure 3) concentrates electromagnetic energy in the dielectric gap between antenna elements. If the gap medium is non-absorbing, which is the case for air, then the field concentrated in the gap will generate no heat.
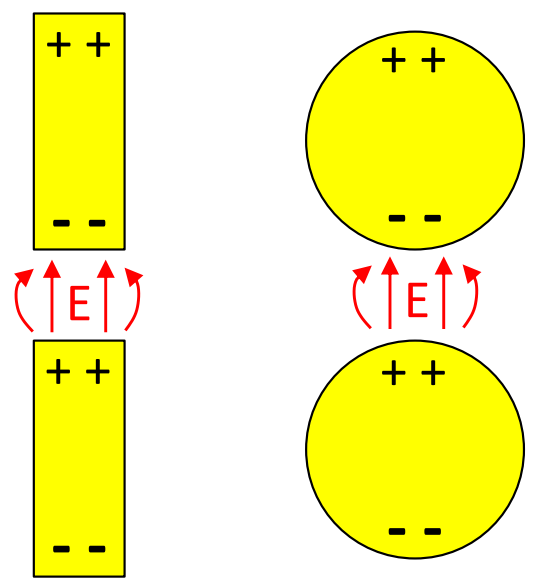

Figure 3. Examples of feed gap antenna geometries.

Other previous thermoplasmonic design studies have looked at the collective effects of particle arrangements. In the first study, spherical particles were arranged on a substrate in a periodic array (Figure $4 a)^{27}$. In each particle, the same amount of heat was generated, yet the temperature of the substrate became much larger than in the case of the single particle with the maximum temperature occurring at the center of the array (Figure 
4b). This occurs because every point in space experiences the thermal effect of each individual particle. As more heat sources/particles are added to the system, the collective effects are summed together, which results in an overall temperature rise in the surroundings ${ }^{27}$. This collective heating property is used later in Chapter $\mathrm{V}$ to calculate the temperature distribution around an optically heated thermoplasmonic structure.

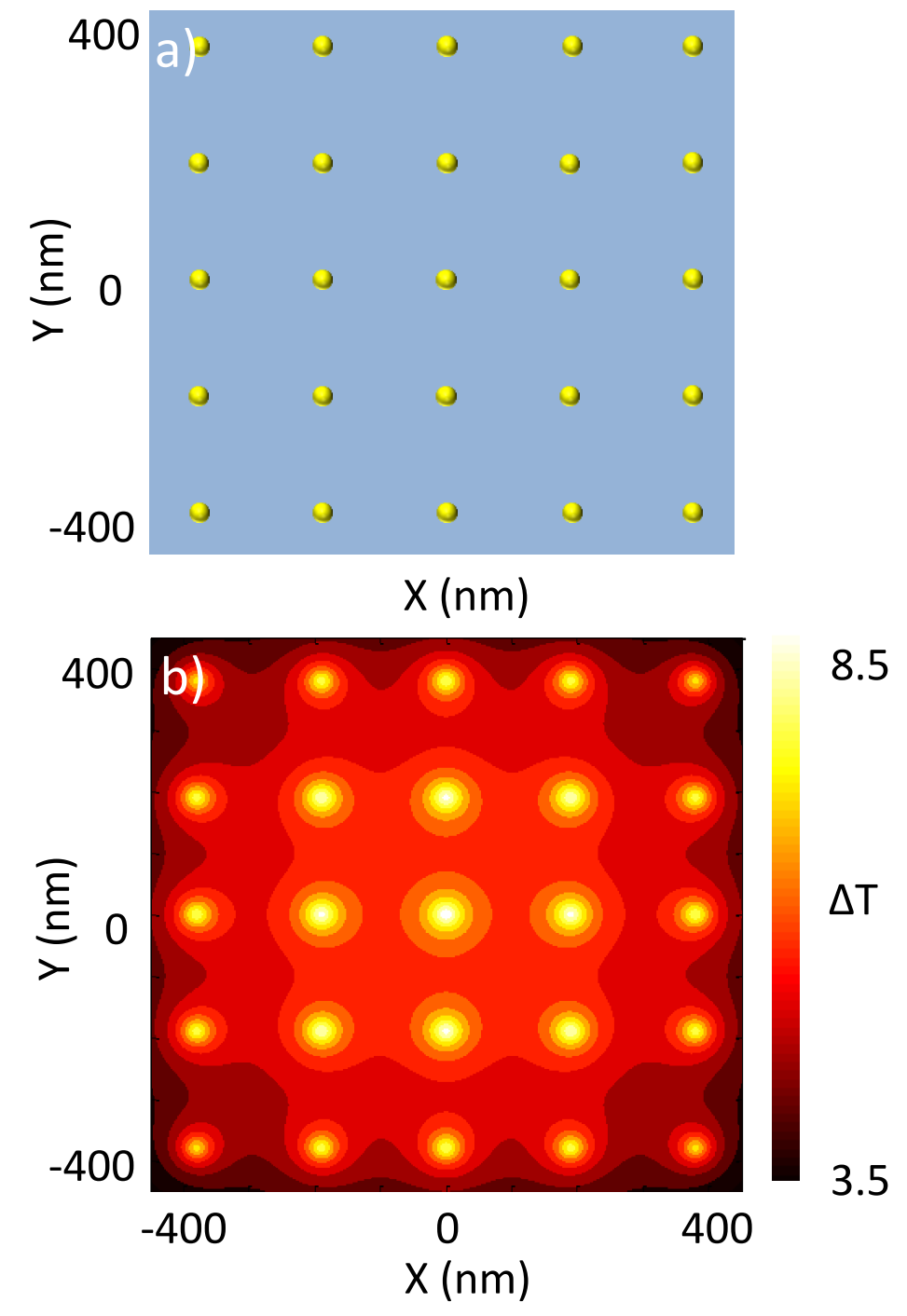

Figure 4. Collective thermal effects of nanoparticle arrangements. a) Layout of $25 \mathrm{~nm}$ spherical particles arranged on a sapphire substrate with a periodicity of $190 \mathrm{~nm} . \mathrm{b}$ ) Temperature distribution of spherical particles at $3 \mathrm{~nm}$ below the sapphire substrate. 
In the second study of particle arrangement, the effects of a three-particle system (two amplifiers and a heater) were analyzied ${ }^{21}$. This three-particle system, called a superstructure, takes advantage of the energy concentration available from plasmonic structures by using two amplifying elements to increase the energy coupled into a heater. In Figure 5, the two larger nanoparticles (amplifiers) electromagnetically couple and generate electric field enhancements across their gap. A smaller particle (heater) placed in the gap readily converts this increased energy input into heat. The results of the study showed a 3.3 times heat generation enhancement when comparing the superstructure to an individual particle ${ }^{21}$. Later in this chapter, a nanoparticle superstructure that more efficiently concentrates electromagnetic energy and generates heat is presented.

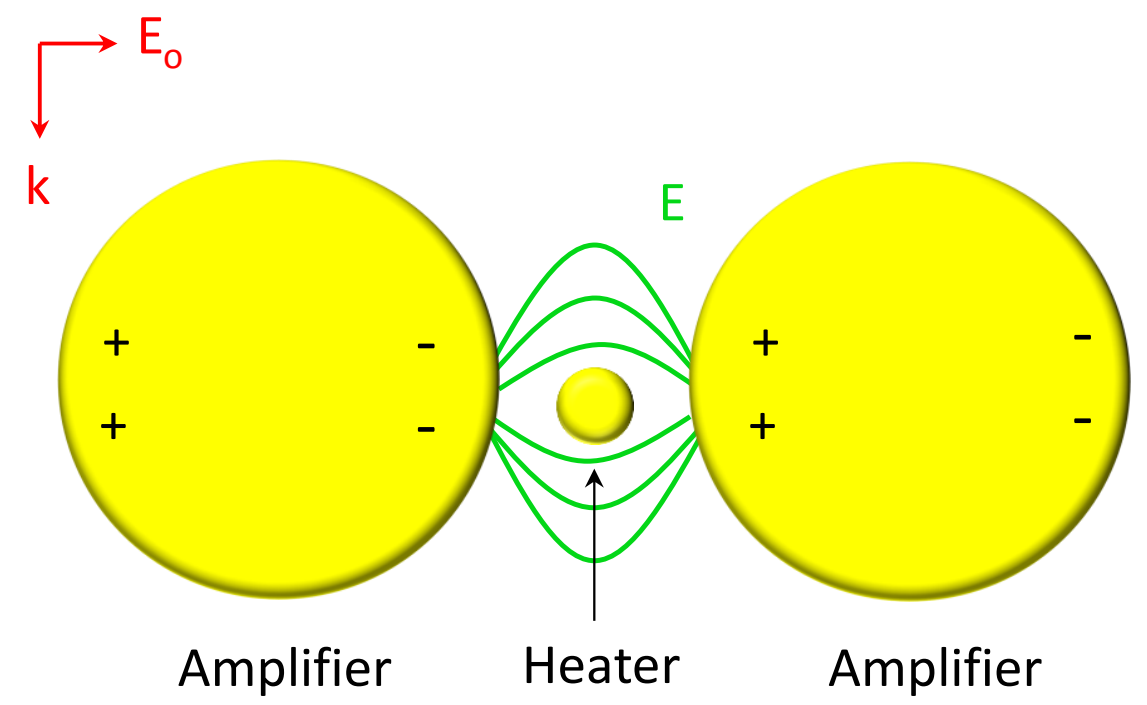

Figure 5. Configuration of plasmonic nanoparticle superstructure. 


\section{Proposed Thermoplasmonic Design Rationale}

The contributions of previous thermoplasmonic design studies have been important to the advancement of the field; yet, in order to fully exploit the potential of thermoplasmonics, a better understanding is needed of how more complex geometries and arrangements can be used to control the magnitude of heat generation in thermoplasmonic nanostructures. To do this, a general understanding of the relationship between heating mechanisms and electromagnetics fields is needed. These relationships are explained in the following sections and used to propose a general design rationale that can be applied to all thermoplasmonic structures.

Heat generation in thermoplasmonic structures is governed by the Joule heating equation, which can be written in terms of current density through Ohm's Law relation:

$$
\begin{gathered}
q^{\prime \prime \prime}=\vec{E} \cdot \vec{J} \\
\vec{J}=\sigma \vec{E} \\
q^{\prime \prime \prime}=\frac{|\vec{J}|^{2}}{\sigma}
\end{gathered}
$$

where $q^{\prime \prime \prime}$ is heat source density $\left[\mathrm{W} / \mathrm{m}^{3}\right], \vec{J}$ is current density, and $\sigma$ is electrical conductivity. As shown in eq 3, optimizing the current density in plasmonic structures will lead to large heat generation values. However, for most nanoparticle design applications, controlling electromagnetic fields rather than current densities is perhaps more intuitive. To show how the magnetic field is related to current density, an application of Ampere's law is used to describe current density as 


$$
\vec{J}=\vec{\nabla} \times \vec{H}
$$

where $\vec{H}$ is the magnetic field ${ }^{28}$. Inserting the relationship for current density into eq 3 allows the heat source density to be expressed as a function of magnetic field,

$$
q^{\prime \prime \prime}=\frac{|\vec{\nabla} \times \vec{H}|^{2}}{\sigma}
$$

If $\vec{J}$ is one dimensional, the only component of $\vec{H}$ is the rotational magnetic field $H_{\theta}$ and therefore,

$$
q^{\prime \prime \prime} \propto H_{\theta}{ }^{2}
$$

The proportionality of eq 6 shows that enhancing the magnetic field around a nanostructure can significantly increase heat generation inside the metal when absorption is dominated by ohmic loss. This finding provides a simple design rationale that is proposed and proven in this thesis.

\section{Enhanced Thermoplasmonic Antenna Design}

One concept for enhancing the magnetic field around metal nanoparticles is the use of complementary structures ${ }^{29,30}$. Based on Babinet's principle for perfect metals, if a structure is complementary (metal and dielectric switch locations) and excited with inverted polarization (rotated by $90^{\circ}$ ), a complementary optical response can be expected. A non-rigorous form of this principle can also be applied to quasi-complementary structures. In these structures, only partial quantities of the dielectric or metal are switched. These quasi-complementary structures still show a complementary optical response, though the response usually does not occur at the same wavelength. 
In this work, the non-rigorous form of Babinet's principle was applied to create the complementary optical response of a feed gap, bowtie antenna. The bowtie antenna, which greatly enhances the electric field through capacitive effects across its gap, was inverted to create a diabolo antenna, which enhances the magnetic field through charge funneling in its neck region ${ }^{29,30}$. Figure $6 a, b$ shows the bowtie and diabolo quasicomplementary structures. Figure $6 \mathrm{c}, \mathrm{d}$ shows plots of the bowtie antenna electric field and the diabolo antenna magnetic field respectively.

a)
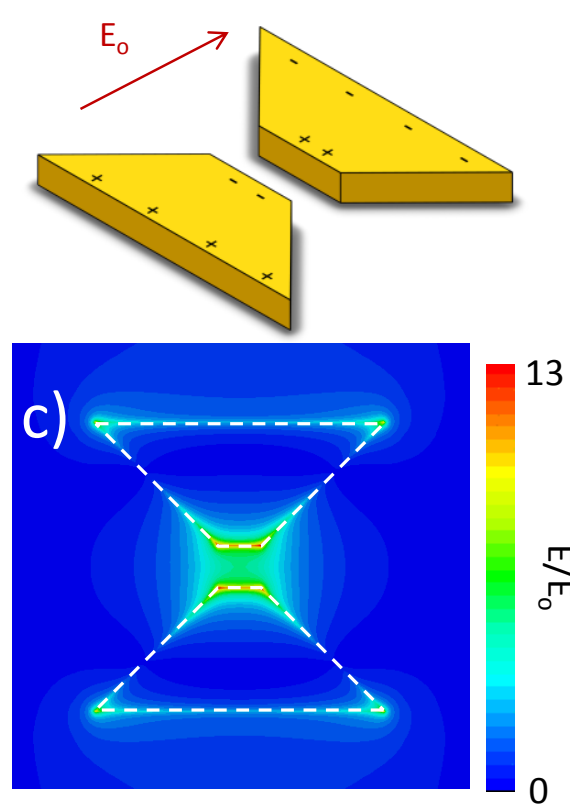

b)

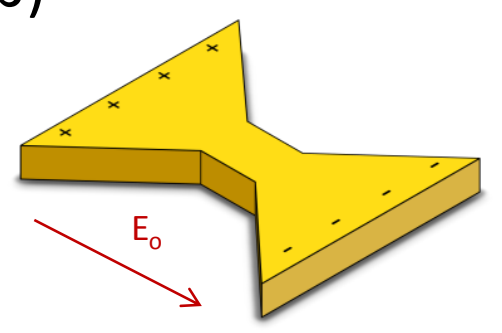

3

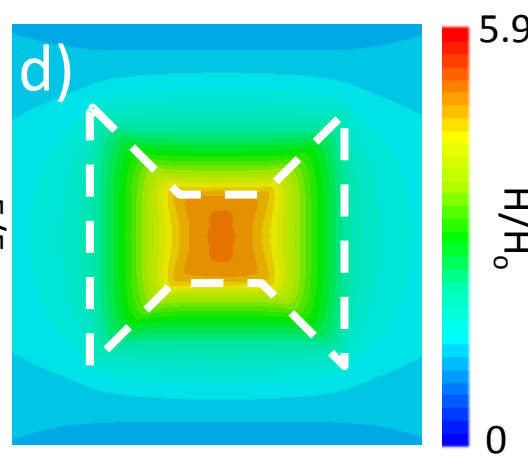

Figure 6. Complementary structures: bowtie (a) and diabolo (b). Plots of electric field (c) and magnetic field (d) are taken at $2 \mathrm{~nm}$ above nanoparticle surface.

Using the diabolo structure as a reference, the proposed thermoplasmonic design rationale of eq 6 was tested against other antenna designs with lesser magnetic field 
enhancements. In Figure 7, the simulated optical and thermal response is shown for an evolution in nanoantenna geometries from the diabolo antenna (flare angle: $45^{\circ}$ ) to a dipole antenna (flare angle: $90^{\circ}$ ). In the evolution, the progressively smaller flare angle of each geometry funnels more charge through the structure's neck region, an effect that increases current density and rotational magnetic field $\left(H_{\theta}\right)$. Probes aligned to the central, vertical axis of each antenna were place at $5 \mathrm{~nm}$ above and $6 \mathrm{~nm}$ inside each structure to capture $H_{\theta}$ and $q^{\prime \prime \prime}$ respectively. Each simulation was performed with a FDTD solver, and the optical properties of the metal were fit to the Drude model ${ }^{31}$. The heat source density was found by inputting the calculated electric field at the specified probe location into the following equation,

$$
q^{\prime \prime \prime}=\pi v \tan \delta \varepsilon_{o} \varepsilon_{m}|E|^{2}
$$

where, $v$ is frequency, $\varepsilon_{\mathrm{m}}$ is metal permittivity, and $E$ is the electric field.

The results from the simulations, presented in Figure 7, show good agreement with the trend expected from eq. 6 . As the rotational magnetic field is increased, the heat source density is correspondingly increased. This result helps to validate the proposed thermoplasmonic design rationale. 


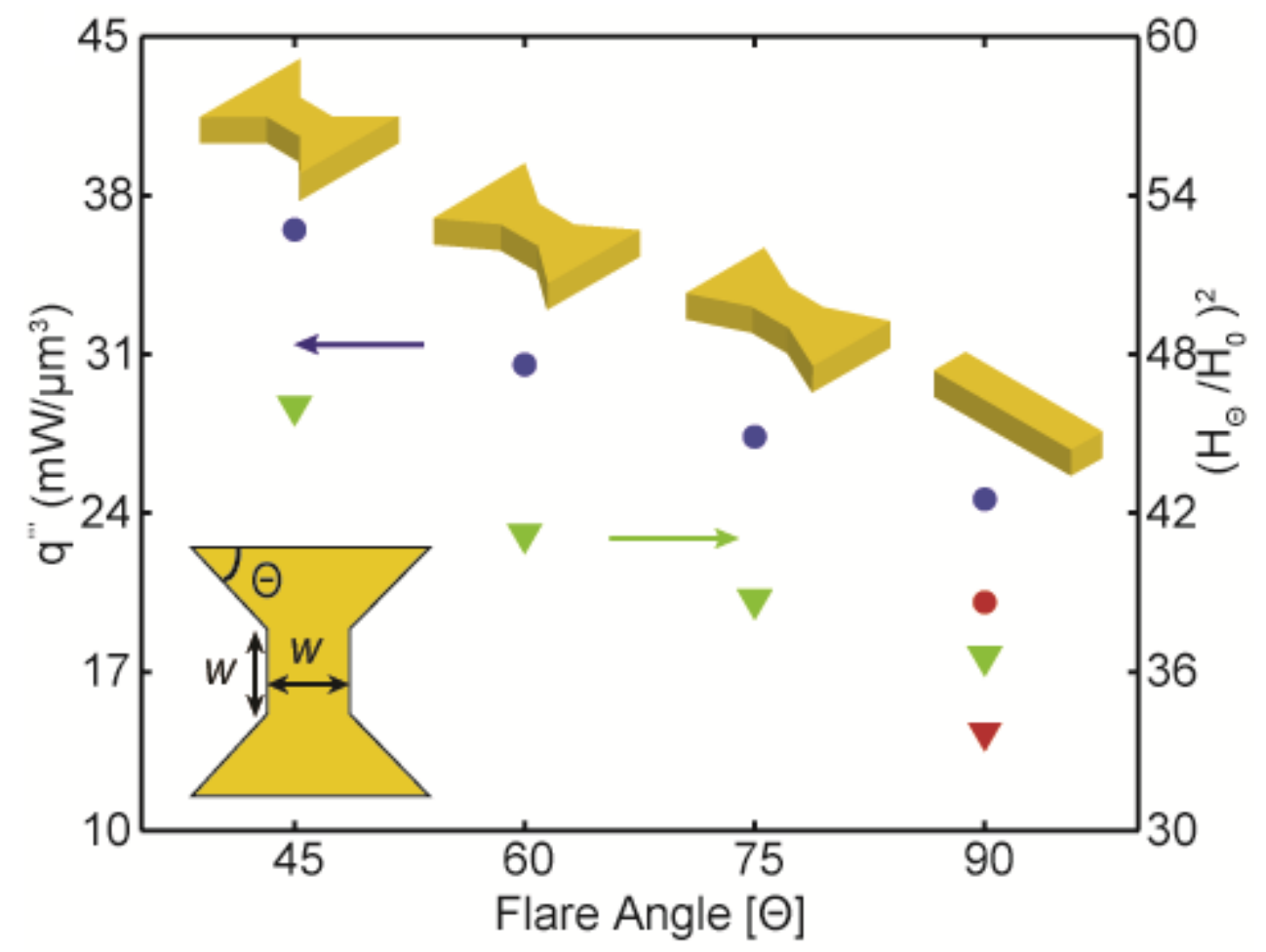

Figure 7. Evolution in geometry from dipole to diabolo antenna ( $w=50 \mathrm{~nm})$. Magnetic fields were normalized to the incoming field intensity and each structure was simulated on an $\mathrm{Al}_{2} \mathrm{O}_{3}$ substrate.

For each structure in Figure 7, the neck dimension $(w)$, resonant wavelength (1064 $\mathrm{nm})$, input intensity $\left(0.42 \mathrm{~mW} / \mu^{2}\right.$ ), and plasmonic material (gold) were held constant to isolate the effects to the varying flare angle. Consequently, equal volumes for each structure could not be maintained. To show that the increase in heat source density was not due to any volumetric effects, a dipole antenna with the same volume, resonant wavelength, and input intensity as the diabolo antenna was simulated. The results show that the dipole structure with a larger volume (red markers in Figure 7) has both a decreased magnetic field and heat source density, confirming that the enhanced heating effect is not volumetric and in fact comes from carefully engineering the antenna's shape. 
As stated previously in the assumptions for eq 6 , the equation is only valid for structures where current is flowing in one direction. To show that the flared geometries presented above have one-dimensional current flow, a proof of concept analysis was performed where the current in an optically excited diabolo nanoantenna was represented with vectors (Figure 8a). From the figure, it is seen that all vectors are directed toward the neck region of the antenna though each vector takes a different angle of approach. Figure $8 \mathrm{~b}$ analyzes two vectors across the symmetric center of the diabolo antenna. Breaking the vectors down into their $x$ - and $y$-components shows that the opposing directions of the two $x$-component vectors cancel one another's contribution while the two $y$-component vectors are added to create an amplified current in the $y$-direction. This analysis shows that one-dimensional flow does in fact exist in the flared geometries, which justifies the application of the design rationale for these structures. 


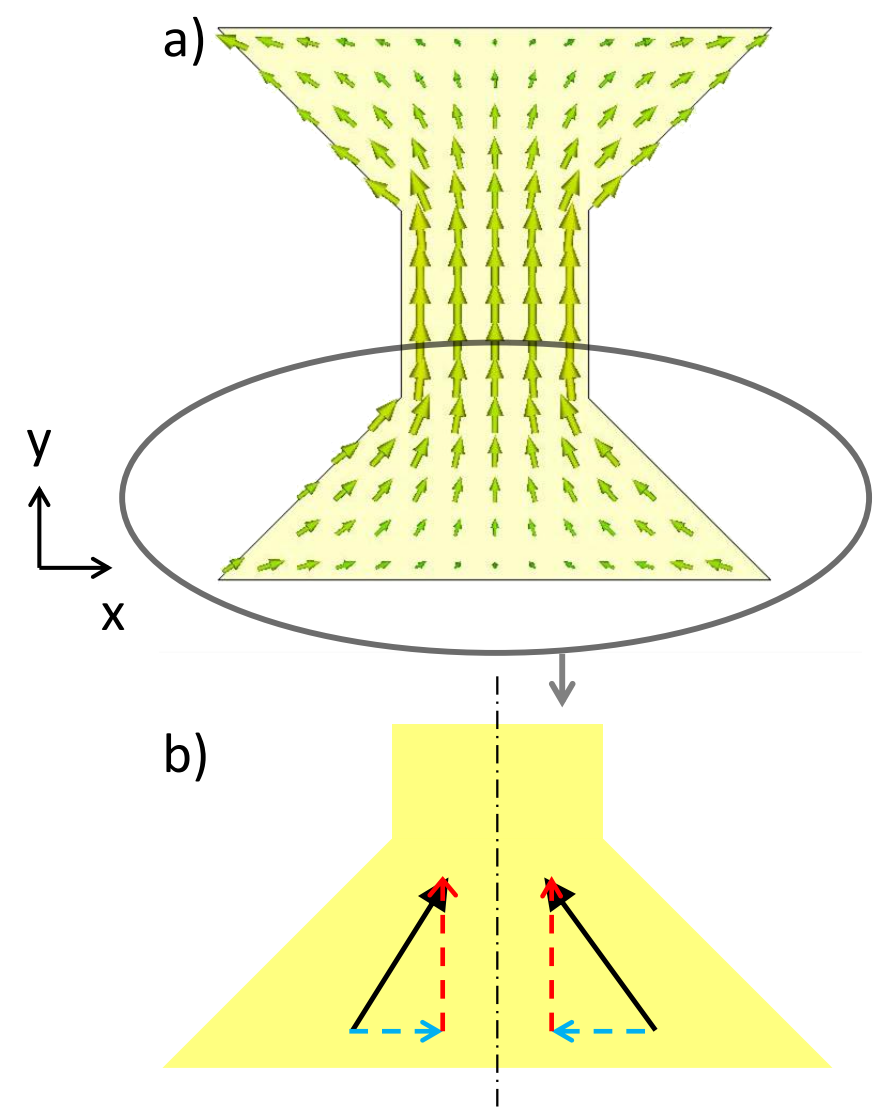

Figure 8. Vectorial representation of current flow in diabolo nanoantenna.

\section{Nanoparticle Superstructure}

The diabolo antenna, as shown in Figure 7, is an optimized thermoplasmonic structure. To take full advantage its enhanced thermoplasmonic design and the non-linear scaling relationship of eq 6 , the antenna was incorporated with a plasmonic bull's-eye lens to create a nanoparticle superstructure. In the superstructure, Figure 9, a lens is used as the amplifier and the diabolo antenna is used as the heater. The lens of the superstructure converts free space light into surface plasmons that are then focused down to the middle ${ }^{32}$. This effect allows for greatly enhanced energy coupling to the diabolo 
antenna while retaining the antenna's nanoscale feature sizes. A superstructure of this design could be used to generate highly localized and enhanced thermal hotspots for applications in heat-assisted magnetic recording or other areas where highly localized and enhanced thermal hotspots are desired.

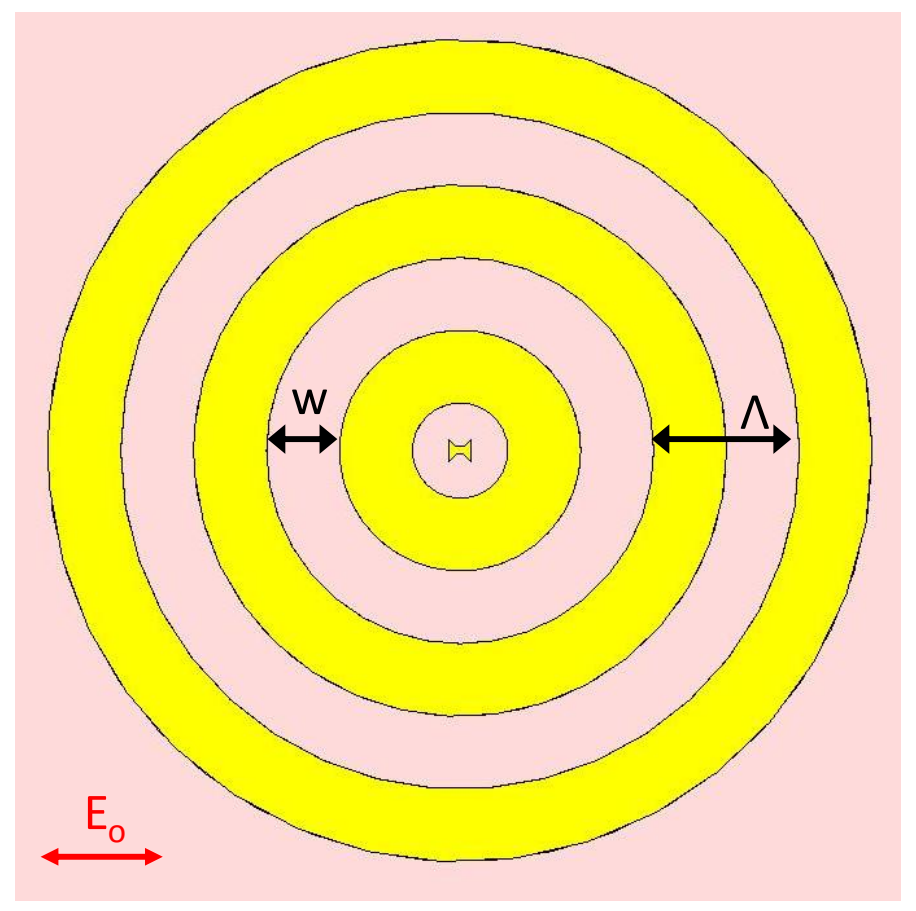

Figure 9. Plasmonic nanoparticle superstructure. $\Lambda$ is the grating constant.

The grating structure of the lens is needed to achieve the focusing effect because surface plasmons do not freely couple to metallic surfaces. This can be shown through the surface plasmon dispersion relation,

$$
R e\left(K_{s p}\right)=\frac{\omega}{c} \sqrt{\frac{\varepsilon_{m} \varepsilon_{d}}{\varepsilon_{m}+\varepsilon_{d}}}
$$


where $\varepsilon_{m}$ is the dielectric function of the metal, $\omega$ is frequency, $K_{s p}$ is the surface plasmon wave vector, and $\varepsilon_{d}$ is the dielectric constant of the dielectric medium. The permittivity of the metal is determined by the Drude model, which is written as,

$$
\varepsilon_{m}(\omega)=1-\frac{\omega_{p}{ }^{2}}{\omega(\omega-i \gamma)}
$$

where $\omega_{p}$ is the plasma frequency and $\gamma$ is the collision frequency. With eq 8 and eq 9 it can be shown that the $K_{s p}$ will never equal $\omega / \mathrm{c}$, the free space wave vector. This is illustrated in Figure 10, which shows a surface plasmon dispersion curve for gold plotted with the light line $(k=\omega / c)$. At every frequency, the $\mathrm{k}$-vector of the air is smaller than that of the surface plasmon. Because of this, schemes to add additional momentum, or kvector, are necessary to couple and focus surface plasmons.

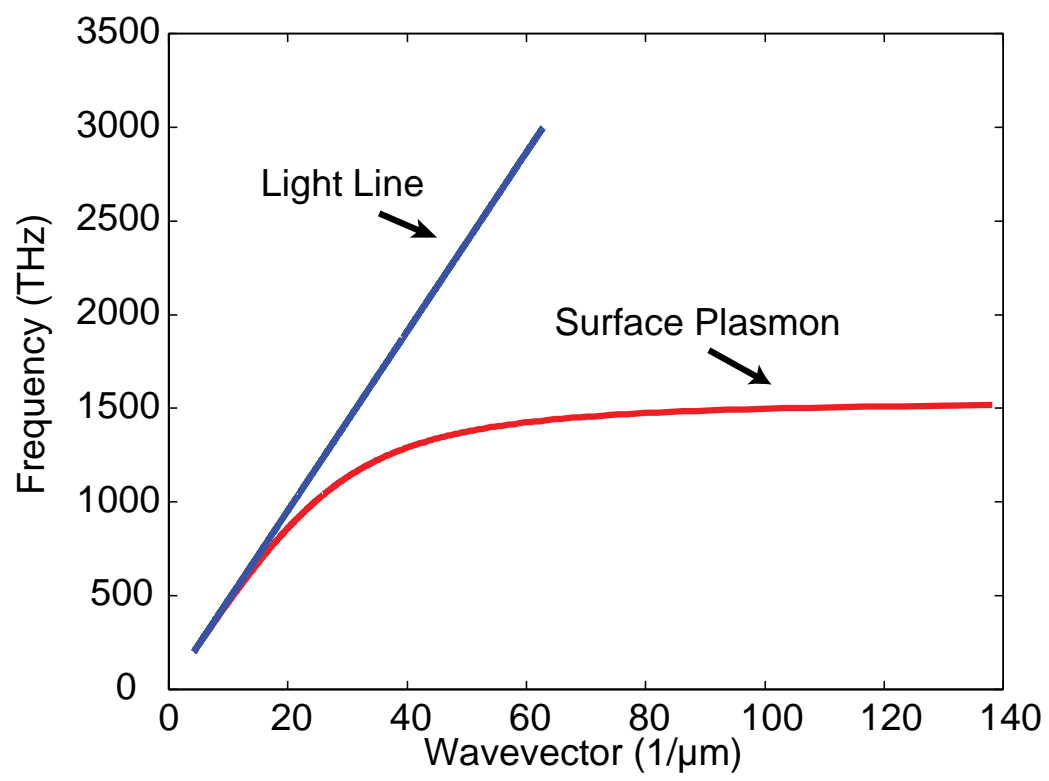

Figure 10. Surface plasmon dispersion curve plotted with the light line. 
The grating is a popular scheme to add momentum at the surface of a metal and dielectric interface. Equation 10 describes the relationship between the surface plasmon $\mathrm{k}$-vector and the dimensions of a 1-D grating,

$$
R e\left(K_{s p}\right)=K_{x}+m \frac{2 \pi}{\Lambda}
$$

where $\mathrm{K}_{\mathrm{x}}$ is the $\mathrm{x}$-component of the incoming $\mathrm{k}$-vector, $\mathrm{m}$ is the diffraction order of the grating, and $\Lambda$ is the grating constant. The first term on the right hand side of eq 10 represents the contribution of $\mathrm{x}$-directed momentum from incoming radiation and the second term represents the additional $\mathrm{x}$-directed momentum added by the grating. Using a diffraction order parallel to the grating surface $(m=1)$ and normally incident light, eq 10 reduces to,

$$
\operatorname{Re}\left(K_{s p}\right)=\frac{2 \pi}{\Lambda}
$$

With eq 11 it is possible to determine the value of a grating constant for any specified surface plasmon k-vector. This is important for the design of the superstructure in Figure 9 as the focused surface plasmon wave vector should match the resonant wave vector of the single diabolo antenna. Using FDTD simulations and the relationship in eq 8 , the resonant wave vector of the single diabolo antenna was found to be $5.59[1 / \mu \mathrm{m}]$. Though eq 11 rigorously applies to 1-D gratings, the bull's-eye shape of the superstructure grating introduces additional complexity that the equation does not capture. As a result, the resonant wave vector of the single diabolo was input to eq 11 to calculate an estimated grating constant for the superstructure. Then, using this estimated value as a basis, a parameter sweep of grating constants in the FDTD simulation was performed to find the $\Lambda$ value that coupled in the most energy $(953 \mathrm{~nm})$. The slit width for the grating in the 
superstructure $(w)$ was set to be half the grating constant or half the surface plasmon wavelength. This was done to reduce any phase accumulation of the plasmon wave and promote constructive interference at the center of the lens. Figure 11 shows a plot of the superstructure's magnetic field. As expected, the magnetic field is enhanced over a single diabolo antenna (compare to Figure 6b). The results from the simulation of the superstructure were incorporated with a thermal model to determine the enhanced heating effects of the structure. Details of the thermal modeling techniques and results are presented in Chapter 5.

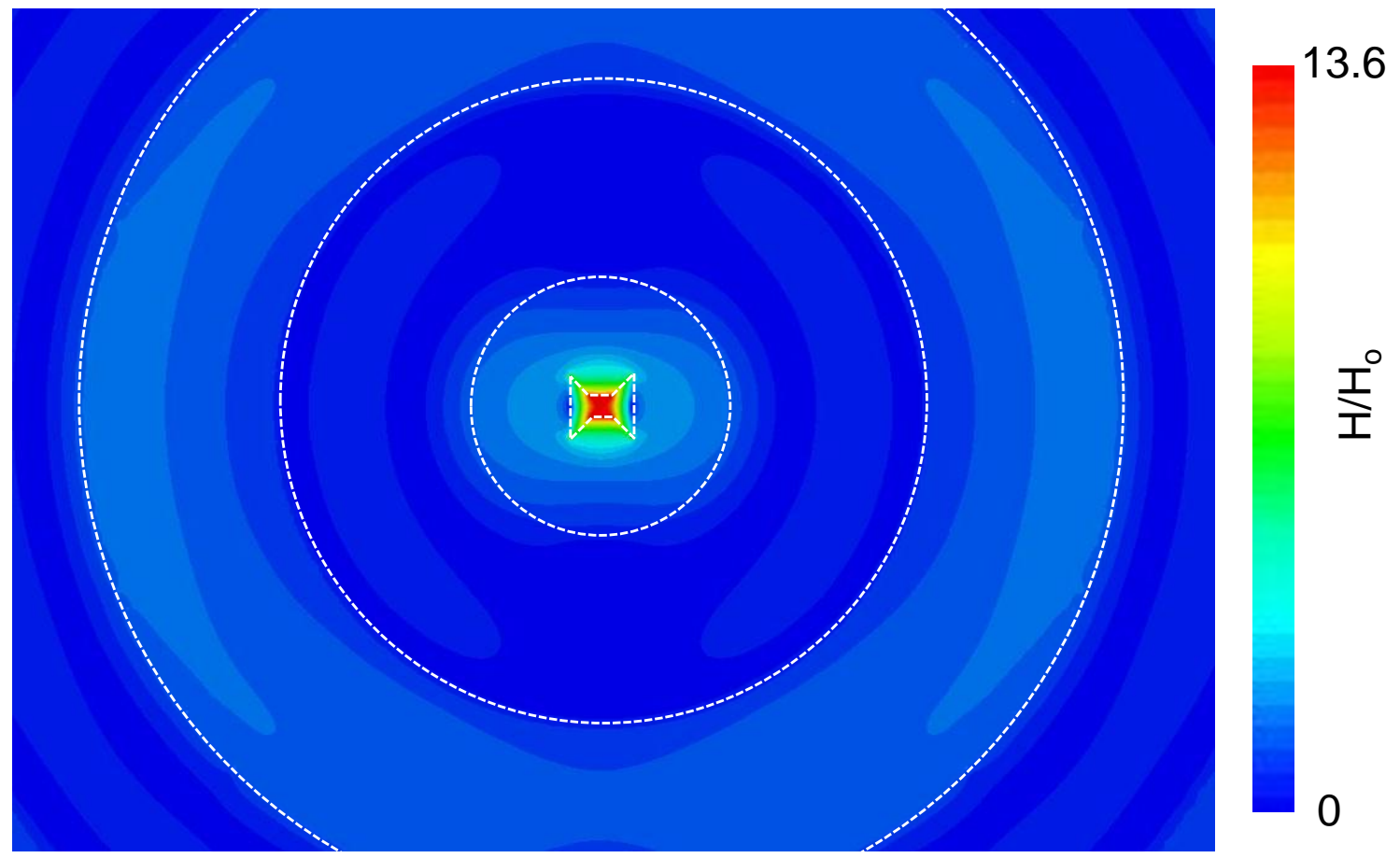

Figure 11. Magnetic field profile of superstructure. 


\section{Conclusion}

The derivations and simulations presented in this chapter provide evidence for the proposed thermoplasmonic design rationale. However, in order to conclusively prove its validity, experimental measurements are needed. Because heat source density is proportional to temperature through the heat diffusion equation,

$$
-q^{\prime \prime \prime}=k \nabla^{2} T
$$

the design rationale can be confirmed through temperature measurements of thermoplasmonic structures with different magnetic field enhancements, such as the dipole and diabolo antenna. In the next two chapters, temperature measurements of thermoplasmonic structures are discussed. In Chapter 3, a new method for measuring micro/nanoscale temperatures is presented along with previous work in this area. In Chapter 4, dipole and diabolo nanoantenna arrays are fabricated, optically heated, and thermally probed. 


\section{CHAPTER III}

\section{MICRO/NANOSCALE TEMPERATURE MEASUREMENTS}

The rise in micro/nanotechnology applications and the recent advances in nanofabrication have driven the need to characterize thermal transport at extremely small scales. As with any thermal characterization, temperature is a very important parameter to measure. Consequently, extensive research over the past twenty years has been devoted to solving the issues associated with micro/nanoscale temperature measurements, as conventional methods do not apply ${ }^{22}$. Though several measurement techniques have been proposed, no one technology has seen widespread adoption. In this chapter, advantages and disadvantages of three existing methods are presented. Then, a new technique involving thermographic phosphors (TGP) is proposed and mechanisms for TGP micro/nanoscale temperature measurements are fully described.

\section{Existing Methods}

Current micro/nanoscale temperature methods can be broken up into two categories: near-field or far-field. Near-field methods are mainly comprised of a technique called scanning thermal microscopy ${ }^{33}$. This method uses a bi-metallic tip to scan a surface of interest, as shown in Figure 12. At the junction of the bimetallic tip, a temperature dependent voltage is generated, much like a thermocouple. A readout system 
is used to measure the voltage change and correlate it with a temperature. The technique offers sub-100 $\mathrm{nm}$ resolution; however, the serial scan process is slow and the method is thermally invasive as the probe can conduct heat away from the surface ${ }^{34}$.

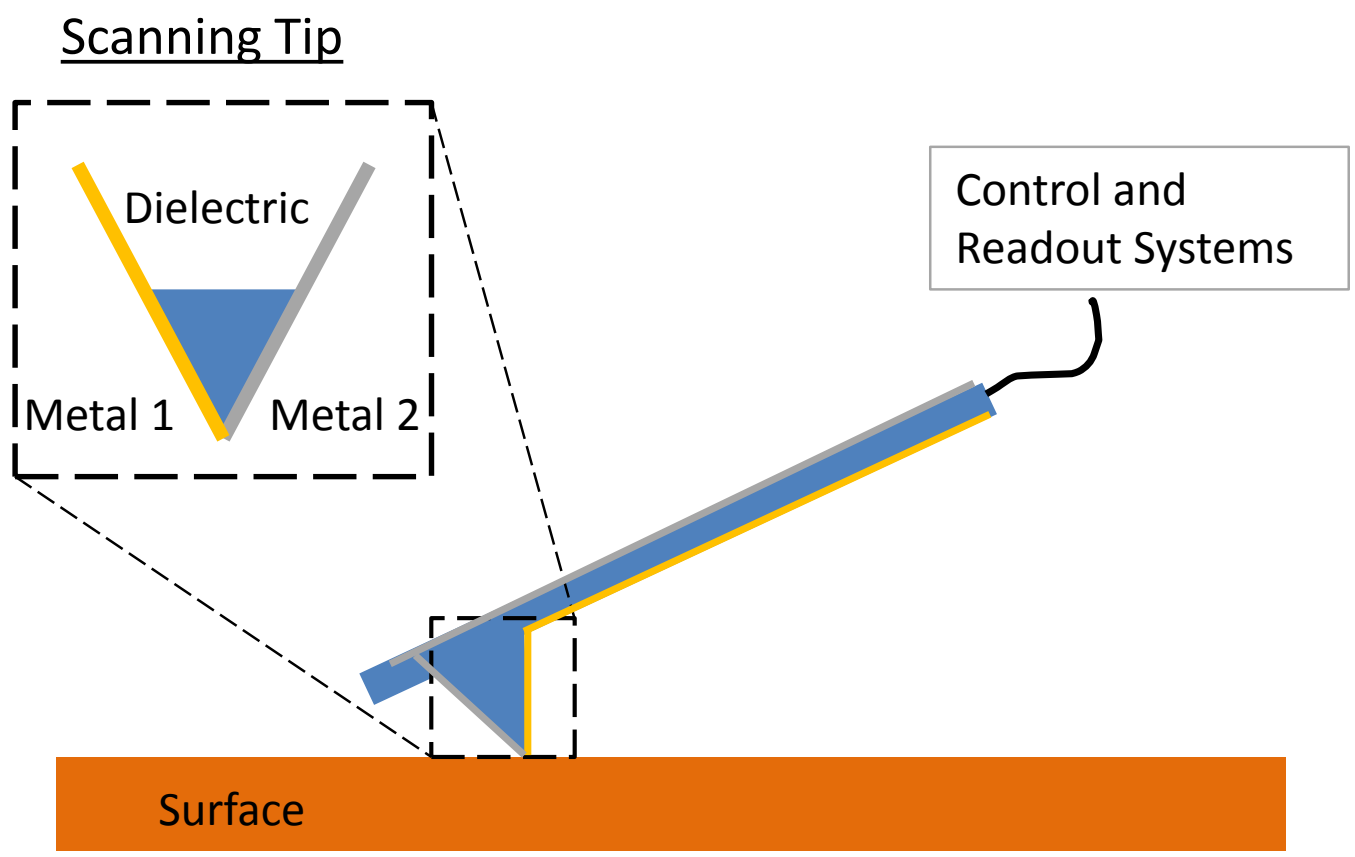

Figure 12. Scanning thermal microscopy schematic.

In the far-field measurement scheme, several techniques exit. One promising approach is based on fluorescence polarization anisotropy ${ }^{4,35}$. In this system, fluorophore molecules are dispersed in an aqueous solution, which is drop cast onto a surface of interest. As the fluorescent molecules increase in temperature, their rotational speed increases. By exciting the fluorophores and measuring their emission intensity in two opposite polarizations, the rotational speed and corresponding temperature can be 
determined. This method can offer roughly $350 \mathrm{~nm}$ resolution at a fast read-out rate. The problem, however, is that at elevated temperatures the liquid solution could evaporate and the emitters could begin to diffuse due to convective currents in the solution. These two issues limit the experimental temperature range of this technique.

Another existing far-field method uses a solid sensing material ( $\left.\mathrm{AlGaN}: \mathrm{Er}^{3+}\right)$ placed near a thermoplasmonic particle to measure temperature ${ }^{36}$. In this scheme, an excitation source of a single wavelength is used to excite both a resonance in a plasmonic particle and the $\mathrm{Er}^{3+}$ atoms in the sensing material. The intensity ratio of two temperature dependent emission peaks of the $\mathrm{Er}^{3+}$ atom is then recorded and used to determine local temperatures. In the study, the group achieved a temperature map around a $40 \mathrm{~nm}$ nanoparticle. The drawbacks from this technique, however, are that the calibration methods are complex, which makes absolute temperatures difficult to obtain, and the technique is based on florescence intensity, which subjects the measurement to errors caused by excitation intensity fluctuations, emitter concentration, and blinking.

\section{Proposed Micro/nanoscale Temperature Measurement Technique}

For the temperature measurements of the thermoplasmonic devices in this thesis, a far-field thermal microscopy technique using thin-film thermographic phosphors (TGP) was developed. TGPs are metal-doped ceramic materials whose photoluminescence decay time is temperature dependent ${ }^{37}$. These materials have long been used to probe temperatures through optical, non-invasive methods and are typically used in 
environments with moving surfaces, high temperatures, or where thermocouple attachment is difficult ${ }^{37,38}$.

To implement this material into a micro/nanoscale temperature measurement system, thin films of TGP were deposited onto a substrate and thermoplasmonic structures were fabricated directly on the film (Figure 13). Then, two beams, one to heat the antenna and one to excite the phosphor, were focused onto the sample. This method allowed for increased accuracy of the temperature measurement due to the sensing layer's close proximity to the antenna. Also, because this technique measures decay lifetimes and uses a solid sensing material (phosphor), it is robust against excitation intensity fluctuations and artifacts due to high surface temperatures, two major issues in existing far-field techniques.

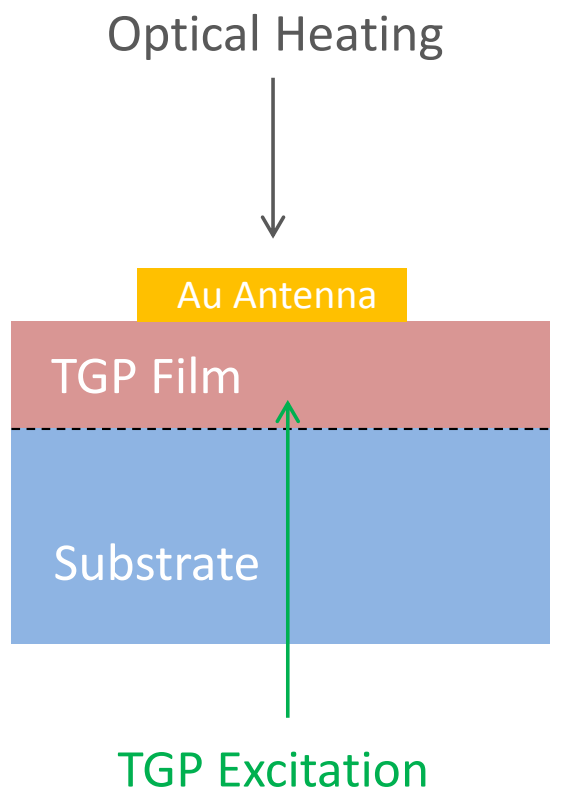

Figure 13. Concept of TGP thermal microscopy technique. Plasmonic antenna is fabricated on phosphor sensing film. An optical source directed from above the antenna induces heating in the structure. An excitation source directed from the below the sample excites the TGP for temperature measurements. 
The general process for determining the temperature of TGPs begins by exciting the electrons of the luminescent dopant atom in the phosphor to higher energy states. Typical excitation sources include electromagnetic radiation, particle beams, and electrical current ${ }^{37}$. After sufficient excitation time, the source energy is removed and the electrons are allowed to relax back to the atom's ground level energy state. The relaxation process occurs in two forms, spontaneous emission and non-radiative relaxation (or thermalization). The rates of each process can be conceptualized as follows:

$$
k=\frac{1}{\tau}=A+W
$$

where $k$ is total decay rate, $\tau$ is total decay time, $A$ is the rate of purely radiative spontaneous emission, and $W$ is the rate of non-radiative relaxation ${ }^{37}$. As the temperature increases in the TGP material, the rate of non-radiative relaxation increases while the spontaneous emission component remains unchanged. This results in a larger total decay rate, $k$, or a smaller decay time, $\tau$. The effect, which can be seen in Figure 14, produces a temperature dependent TGP decay that can be used to measure absolute temperatures through a calibration procedure. 


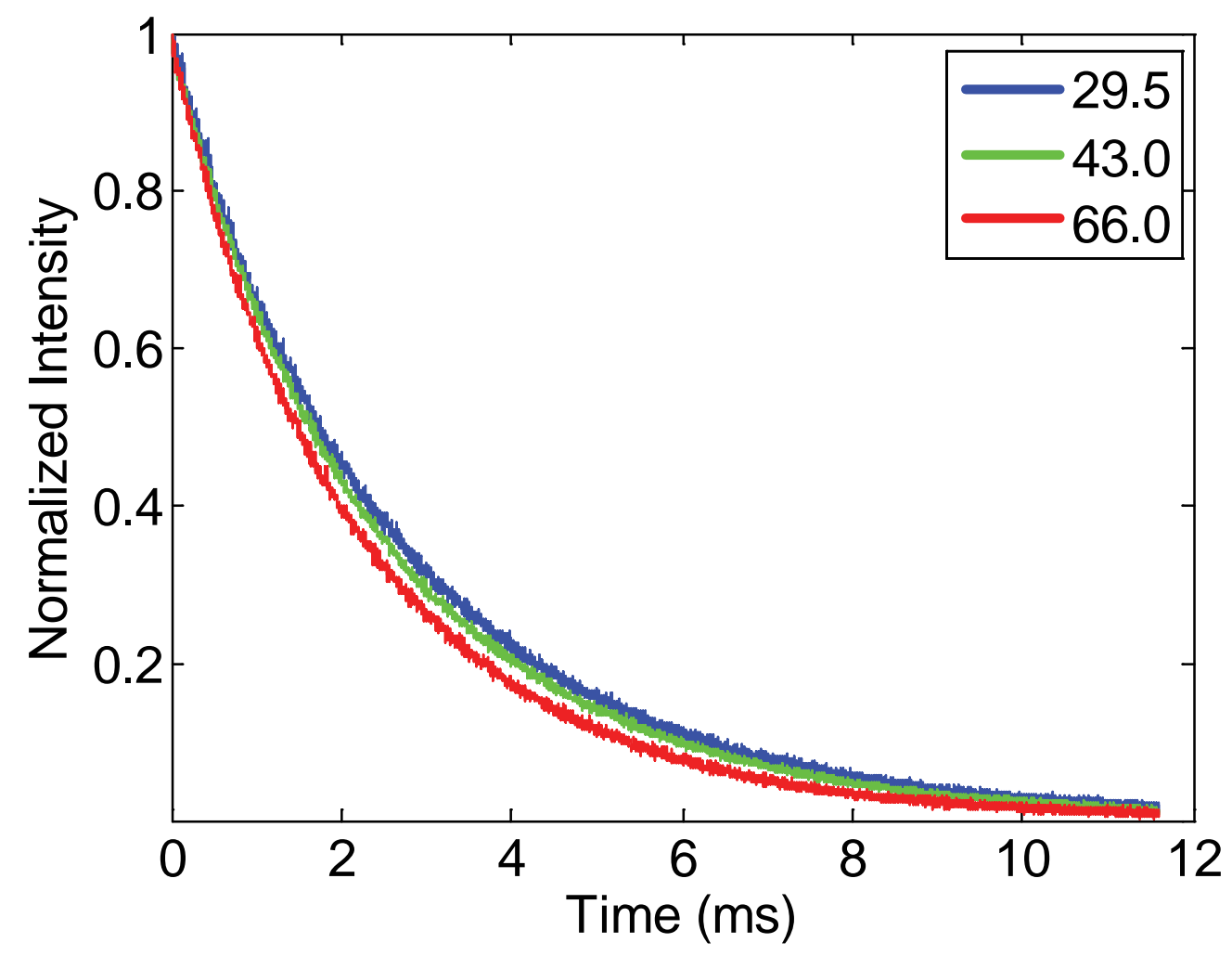

Figure 14. Decay plots for varying phosphor temperatures. Higher temperatures result in faster decay.

During the calibration process, the phosphor temperature is incrementally changed with a heating element and measured using an accurate reference device such as a thermocouple. At each temperature, an intensity vs. time measurement, such as the one presented in Figure 14, is taken. To find the total decay time, the data is fit to an exponential function and solved for $\tau$ :

$$
I(t)=I_{o} e^{-t / \tau}
$$

where $I$ is intensity, $t$ is time, and $\tau$ is decay time ${ }^{37}$. A sample calibration curve for the phosphor lanthanum oxysulfide is shown in Figure 15. Using this model, any decay time taken of a specific phosphor can be mapped to an absolute temperature. 


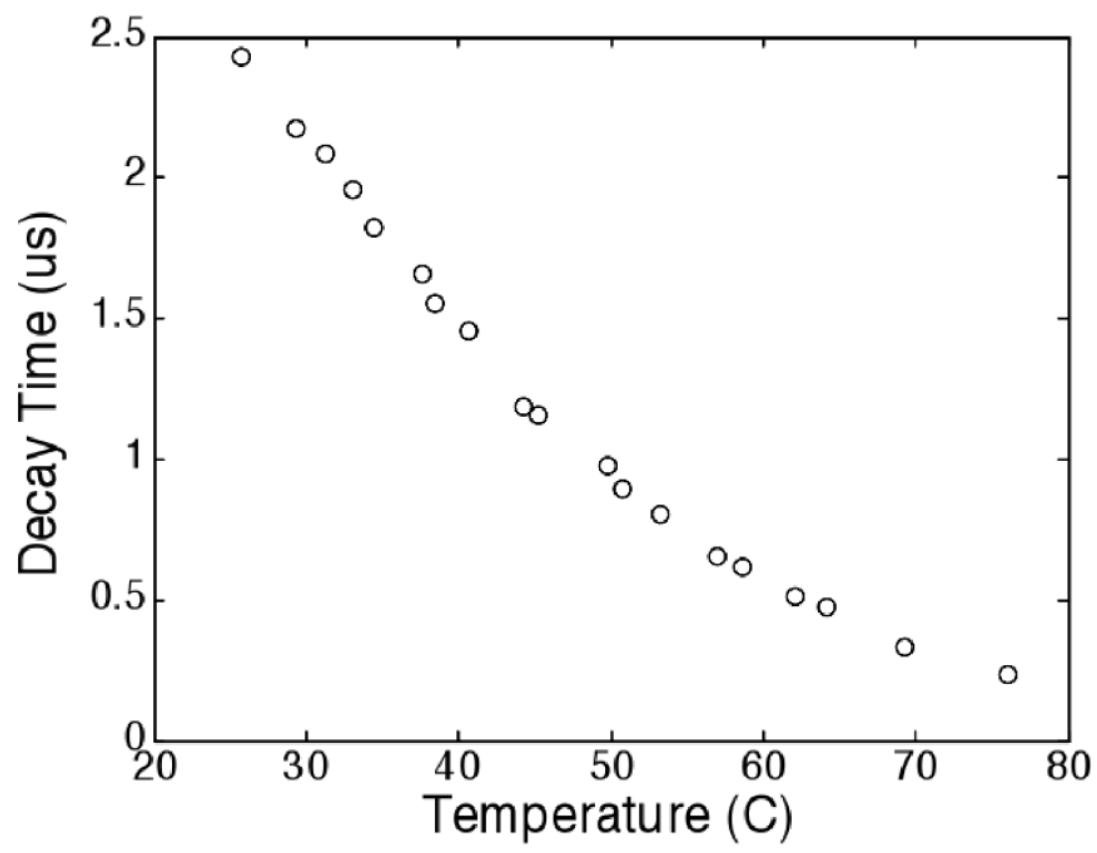

Figure 15. Calibration curve of lanthanum oxysulfide taken at $514 \mathrm{~nm}$ emission peak. 


\section{CHAPTER IV}

\section{METHODS/RESULTS}

In this chapter, the design rationale presented in Chapter 2 is experimentally validated by measuring the temperature of optically heated dipole and diabolo nanoantenna arrays. The temperature measurements are performed with the TGP microscopy technique proposed in Chapter 3. This chapter includes descriptions of the fabrication and characterization processes for both the TGP film and the antenna array, a layout of the TGP measurement scheme, and an overview of the phosphor calibration process. At the end of the chapter, results for the temperature measurements of the dipole and diabolo antenna arrays are reported.

\section{Fabrication}

\section{Thin-film TGP}

All thermoplasmonic temperature measurements in this thesis were performed using the thin-film TGP arrangement in Figure 13. The specific phosphor used in the experiments was ruby $\left(\mathrm{Al}_{2} \mathrm{O}_{3}: \mathrm{Cr}^{3+}\right)$. Ruby was chosen because of its excellent sensitivity over the experimental range and because its temperature dependent emission wavelength $(694 \mathrm{~nm})$ does not overlap the antenna resonant wavelength $(1064 \mathrm{~nm})^{38-40}$.

All fabrication and characterization steps of the thin-film phosphor layer were performed by lab mate Wei Li. In the first step of the process, a ruby TGP powder was 
made using combustion synthesis, Figure 16a. Next, the powder was loaded into an electron beam evaporation chamber and a $100 \mathrm{~nm}$ film of ruby was deposited onto a sapphire substrate, Figure $16 \mathrm{~b}$. To reconstitute the $\alpha-\mathrm{Al}_{2} \mathrm{O}_{3}$ from the as-deposited $\mathrm{Al}_{2} \mathrm{O}_{3}$, the sample was thermally annealed at $1100^{\circ} \mathrm{C}$ for 2 hours. This was an important step in the process as the $\alpha-\mathrm{Al}_{2} \mathrm{O}_{3}$ has stronger photoluminescence properties than the asdeposited phase (Figure 17), and the $\alpha-\mathrm{Al}_{2} \mathrm{O}_{3}$ is the temperature dependent phase of the material $^{38}$. The sapphire substrate was used to allow for perfectly matched thermal expansion coefficients between the film and substrate, thus minimizing film stress and cracking during the annealing process. Any cracks or roughness in the ruby film would affect the fabrication of the thermoplasmonic structures. Figure 18 shows an atomic force microscope (AFM) image of the ruby film after annealing. The $2.4 \mathrm{~nm}$ rms roughness of the film indicates a quality film for antenna fabrication.
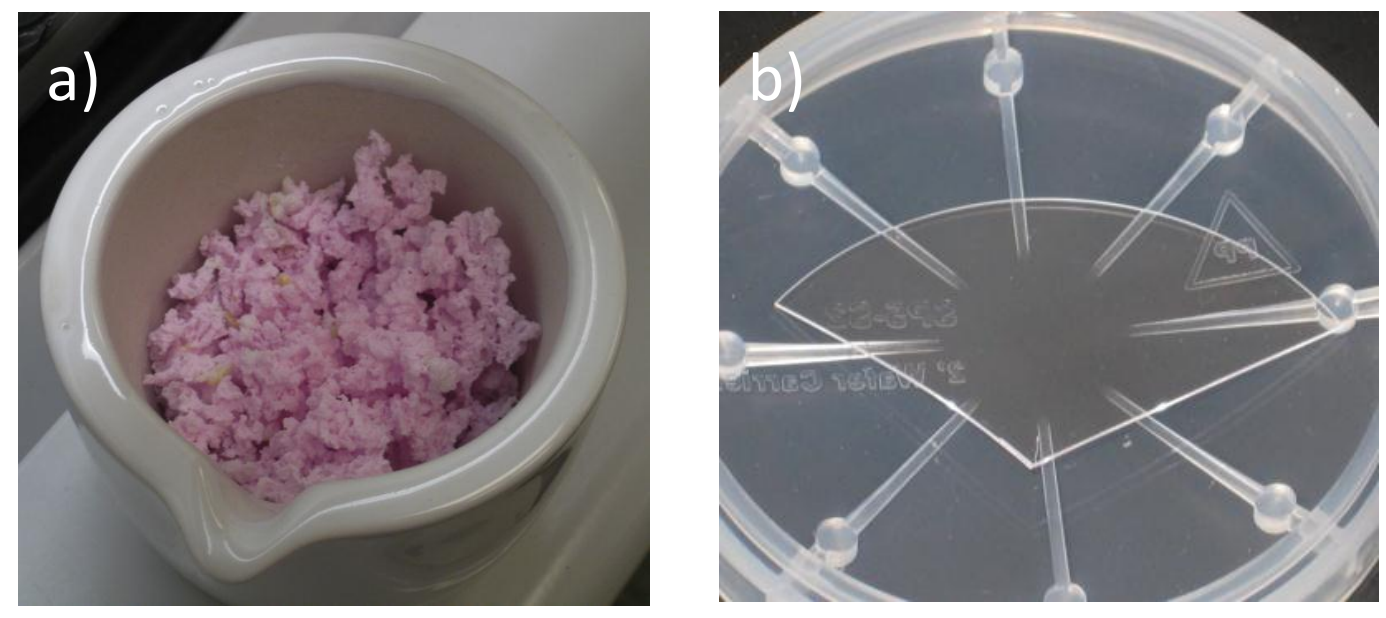

Figure 16. Ruby powder (a) and deposited thin-film (b). 


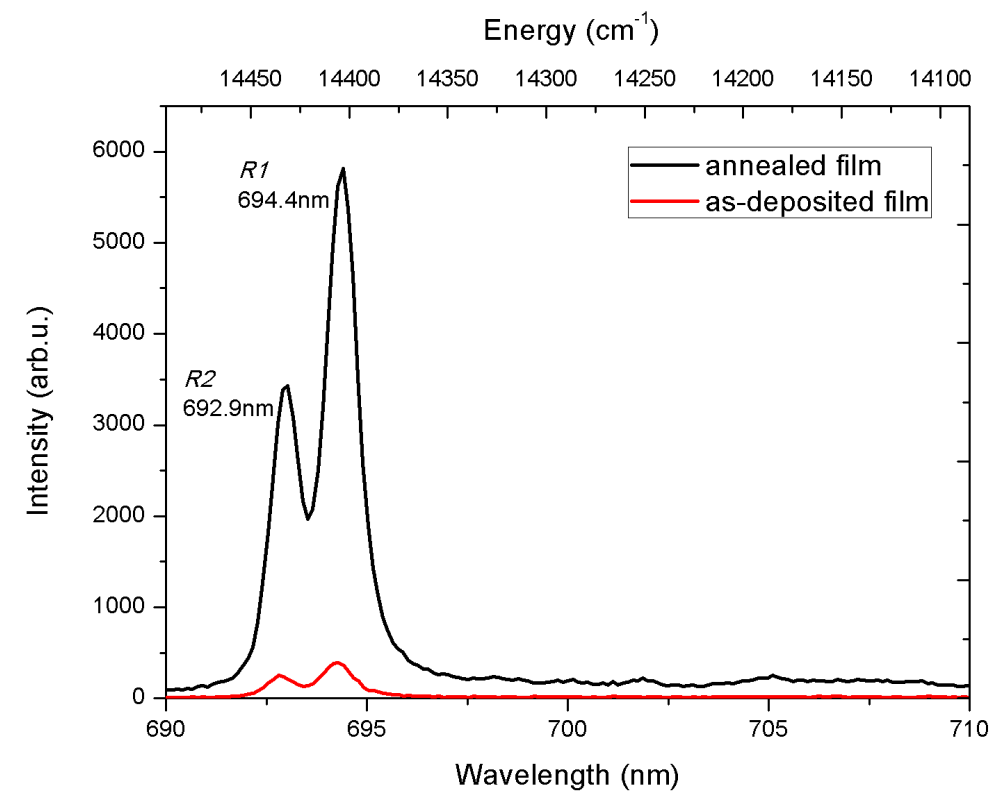

Figure 17. Emission spectra of annealed and un-annealed ruby phosphor films. Excitation of the phosphor was performed with a $532 \mathrm{~nm}$ laser.

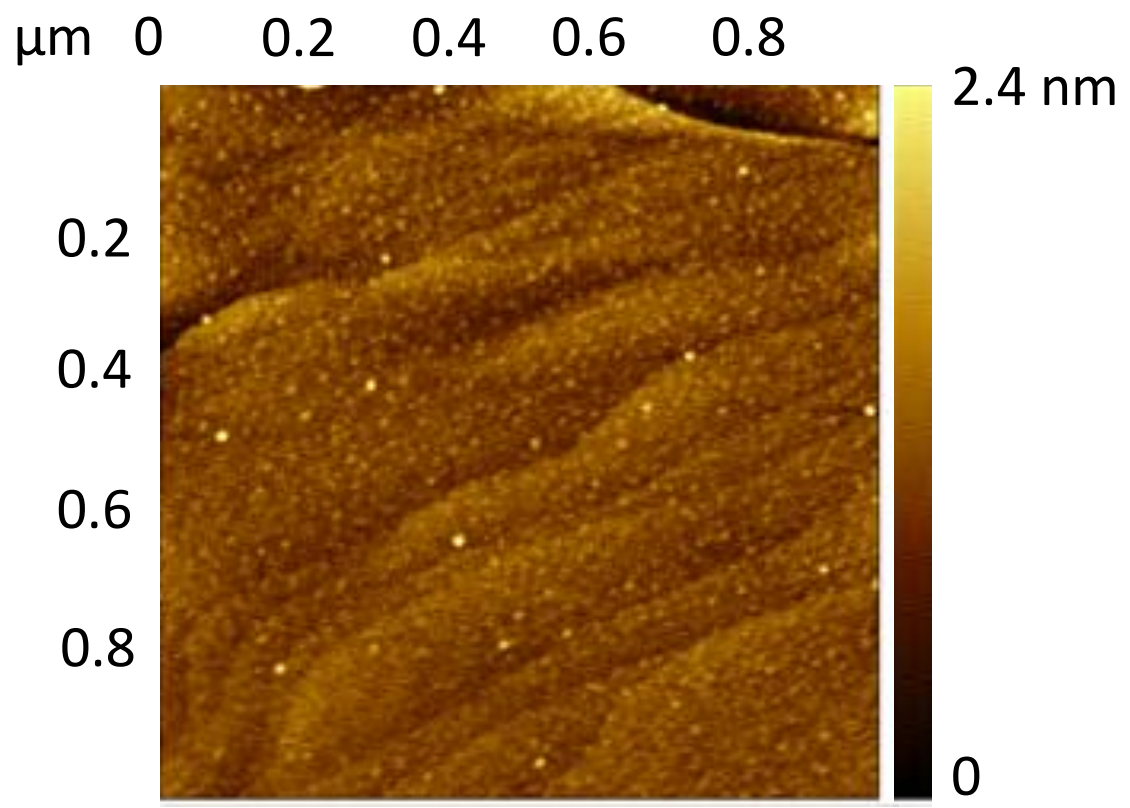

Figure 18. AFM image of deposited ruby film on sapphire substrate. 


\section{Antenna Arrays}

Antenna arrays were fabricated directly on the prepared ruby film using an electron beam lithography (EBL), Cr/Au deposition, and ultrasonic liftoff process. Figure 19 shows the three-dimensional unit cell of the dipole and diabolo antenna array.

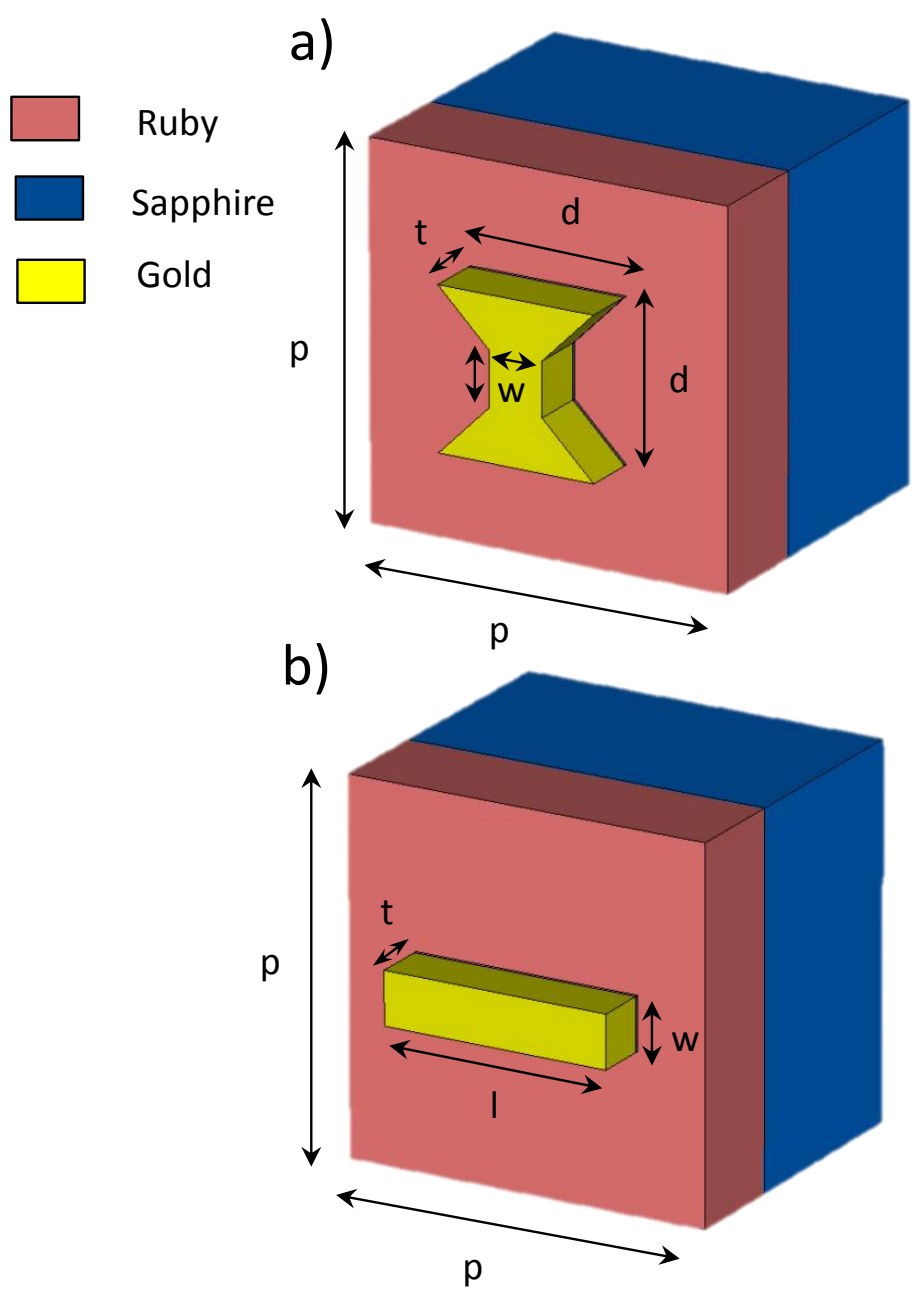

Figure 19. Design of antenna arrays. (a) Unit cell of diabolo antenna array with dimensions: $\mathrm{p}=340 \mathrm{~nm}, \mathrm{~d}=150, \mathrm{w}=50 \mathrm{~nm}, \mathrm{t}=50 \mathrm{~nm}$. (b) Unit cell of dipole antenna array with dimension: $1=215 \mathrm{~nm}$. Each array is made up of 250x250 antenna structures. 
For the EBL process, PMMA-A4 was spin-coated on top of the ruby layer to a thickness of $222 \mathrm{~nm}$ and baked at $180^{\circ} \mathrm{C}$ for 10 minutes on a hot plate. The post-baking process was used to evaporate any solvents in the resist. After the post-bake, the sample was loaded into a Raith EBL tool and exposures of the patterned arrays were performed. Electron beam exposures of a positive photoresist, such as PMMA-A4, break down the polymer chains in the resist for later removal by a "developing" solvent. EBL offers the highest lithography resolution for nanoscale fabrication as it can achieve feature sizes near $10 \mathrm{~nm}$.

Following the EBL exposures, the sample was developed using a cold development process. The cold development process has been shown to increase the resolution of nanosized features by increasing the selectivity of a developer in removing exposed photoresist $^{41}$. The increased selectivity of the developer allows for longer EBL exposures (which increases writing accuracy) and decreased parasitic overexposure effects. For the cold development process in this study, the developer MIBK/IPA 1:3 was placed in an ice bath and held at a temperature of $0^{\circ} \mathrm{C}$. The patterned sample was then placed in this solution for 2 minutes followed by a 30 second rinse in IPA to complete the development process.

After development, the sample was loaded into a thermal evaporation chamber for the deposition of chromium and gold. Three nanometers of chromium was deposited first at a rate of $0.4 \mathrm{~A} / \mathrm{s}$. The chromium was used as an adhesion layer between the gold and ruby substrate. After chromium, $50 \mathrm{~nm}$ of gold was deposited at a rate of $1.5 \mathrm{~A} / \mathrm{s}$. Figure 20 shows a cross-sectional depiction of the sample after the EBL, development, and deposition steps. 


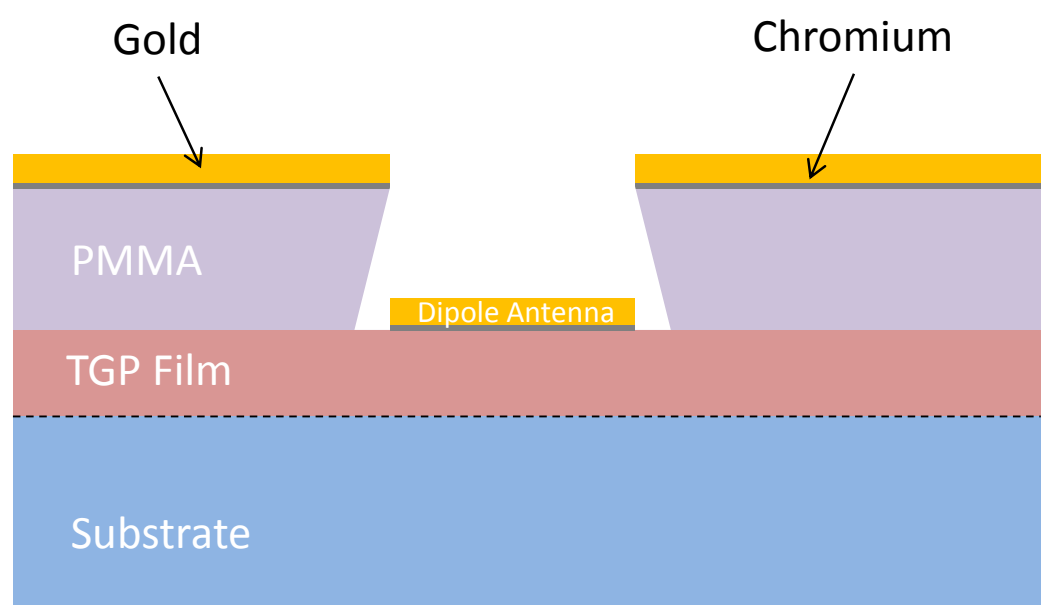

Figure 20. Cross-sectional depiction of sample after EBL exposure and $\mathrm{Cr} / \mathrm{Au}$ deposition.

The final step in the fabrication of the nanoantenna arrays was ultrasonic liftoff of the PMMA. For this step, two beakers were filled with Remover 1165 and placed on a hot plate set to $75^{\circ} \mathrm{C}$. Two more beakers, one filled with IPA and the other with de-ionized (DI) water, were set to the side. After soaking the sample in the Remover 1165 beaker for 2 hours, the beaker was placed in an ultrasonic bath set to $100 \mathrm{kHz}$. After removing most of the PMMA with ultrasonic vibrations, the sample was then placed in the other Remover 1165 beaker on the hot plate and allowed to soak for 1 hour. This extra soak step facilitated the removal of the remaining PMMA on the sample. Following this soak, an IPA and DI water rinse removed any residue left on the surface to complete the lift-off process. Figure 21 shows SEM images of the final fabricated nanoantenna arrays. 


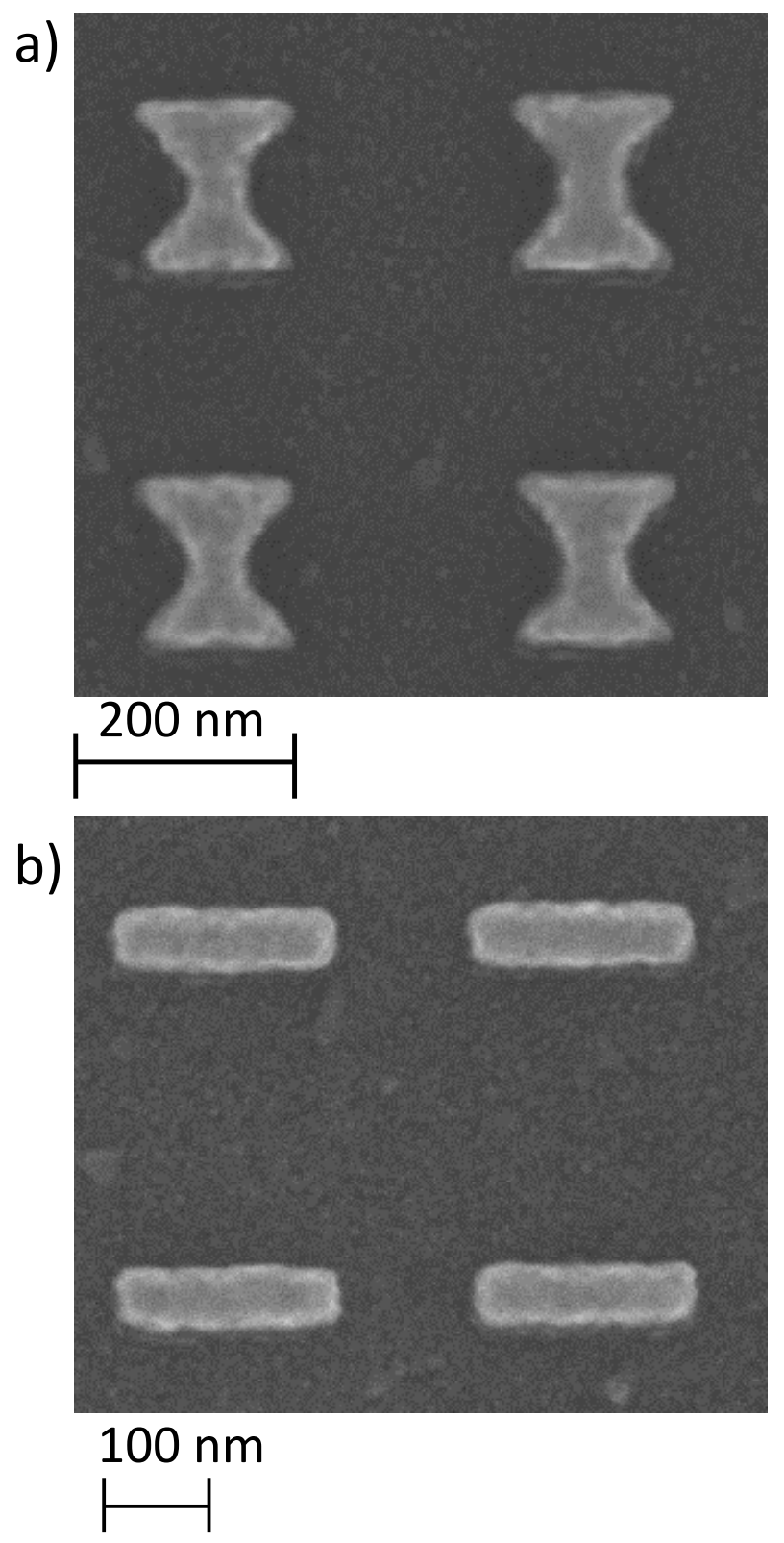

Figure 21. SEM images of the (a) diabolo antenna array and (b) dipole antenna array.

\section{Array Characterization}

Characterization of the arrays was performed to verify that the resonant position of the fabricated structures matched the resonant position of the simulated structures. 
This was done by collecting the transmission data from both arrays. Transmission data is a good indicator of the resonant wavelength owing to the fact that at resonance, surface plasmons strongly absorb and scatter incoming electromagnetic radiation. This absorption and scattering attenuates the light passing through the structure causing a sharp dip in transmission.

Transmission measurements of the dipole and diabolo arrays were taken by directing a broadband, polarized light source through the topside of each array. The transmitted light was collected with an objective focused on the backside of each array and then directed to a spectrometer where the intensity at each wavelength was determined. The light transmitted through the arrays was normalized to the light transmitted through a bare part of the ruby/sapphire substrate. Figure 22a,b shows the simulated and experimental transmission data of the diabolo and dipole arrays. The data show good agreement with the spectral position of the resonant wavelength as the transmission dip in both plots is located near $1064 \mathrm{~nm}$. 

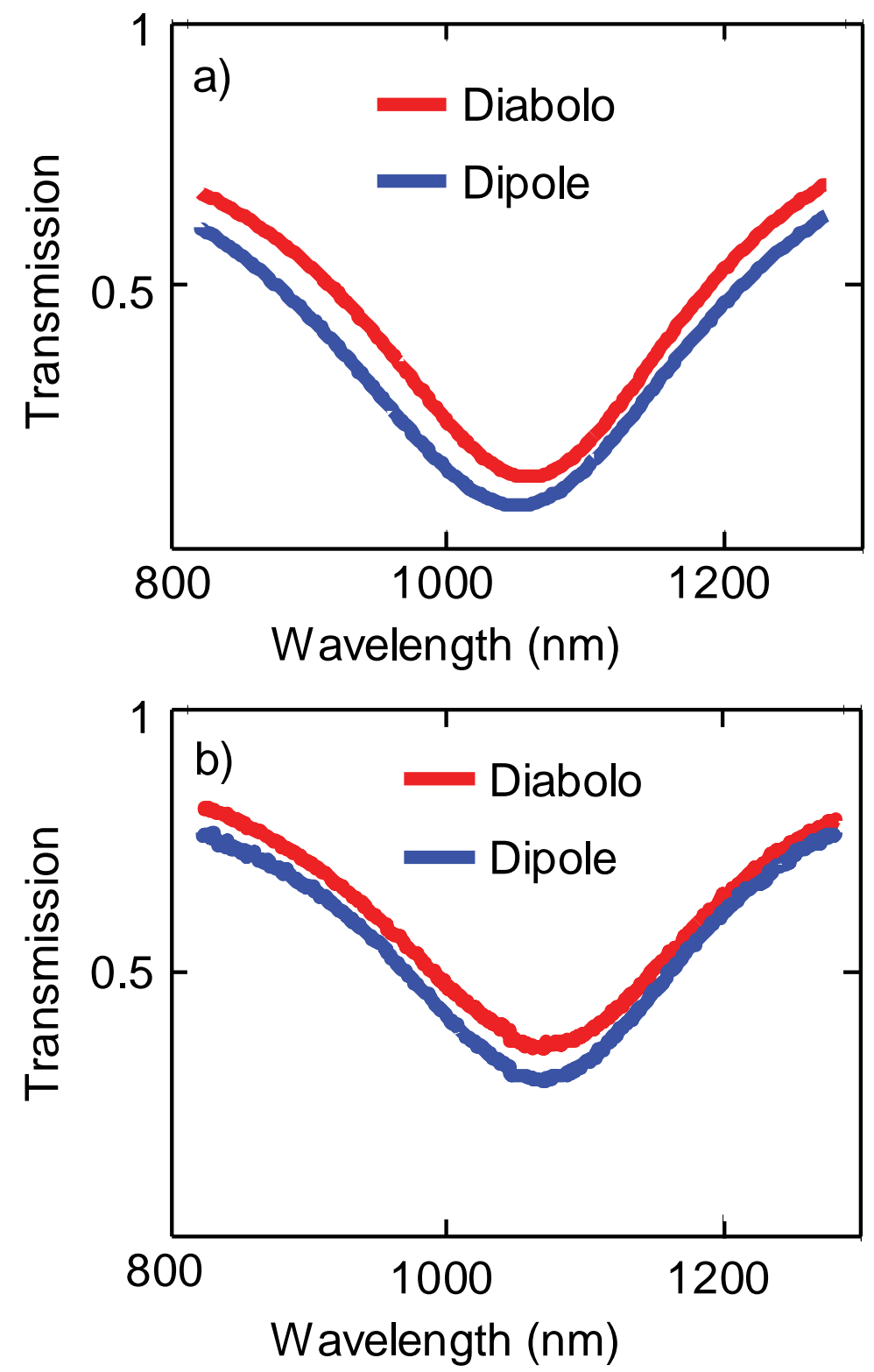

Figure 22. Plot of the (a) simulated and (b) experimental transmission data.

\section{Thermal Microscopy Setup}

The resonant heating and thermal probing of the fabricated samples was performed with the thermal microscopy setup shown in Figures 23 and 24. A Ti:Sapphire pumped 
optical parametric oscillator (OPO) was used to generate the infrared heating beam (1064 $\mathrm{nm})$ and the phosphor excitation beam $(532 \mathrm{~nm})$. Both beams were weakly focused through objectives and spatially overlapped on the sample to achieve simultaneous pump/probe measurements. Though the phosphor excitation beam was pulsed, an optical chopper was used to modulate the beam since the phosphor decay is on the order of milliseconds and the source pulse is on the order of femtoseconds. The chopper was set to rotate at $9 \mathrm{~Hz}$. This rotational rate allowed the excitation beam to fully charge the electrons in the phosphor during the on-state and allowed the phosphor to fully decay during the off-state.

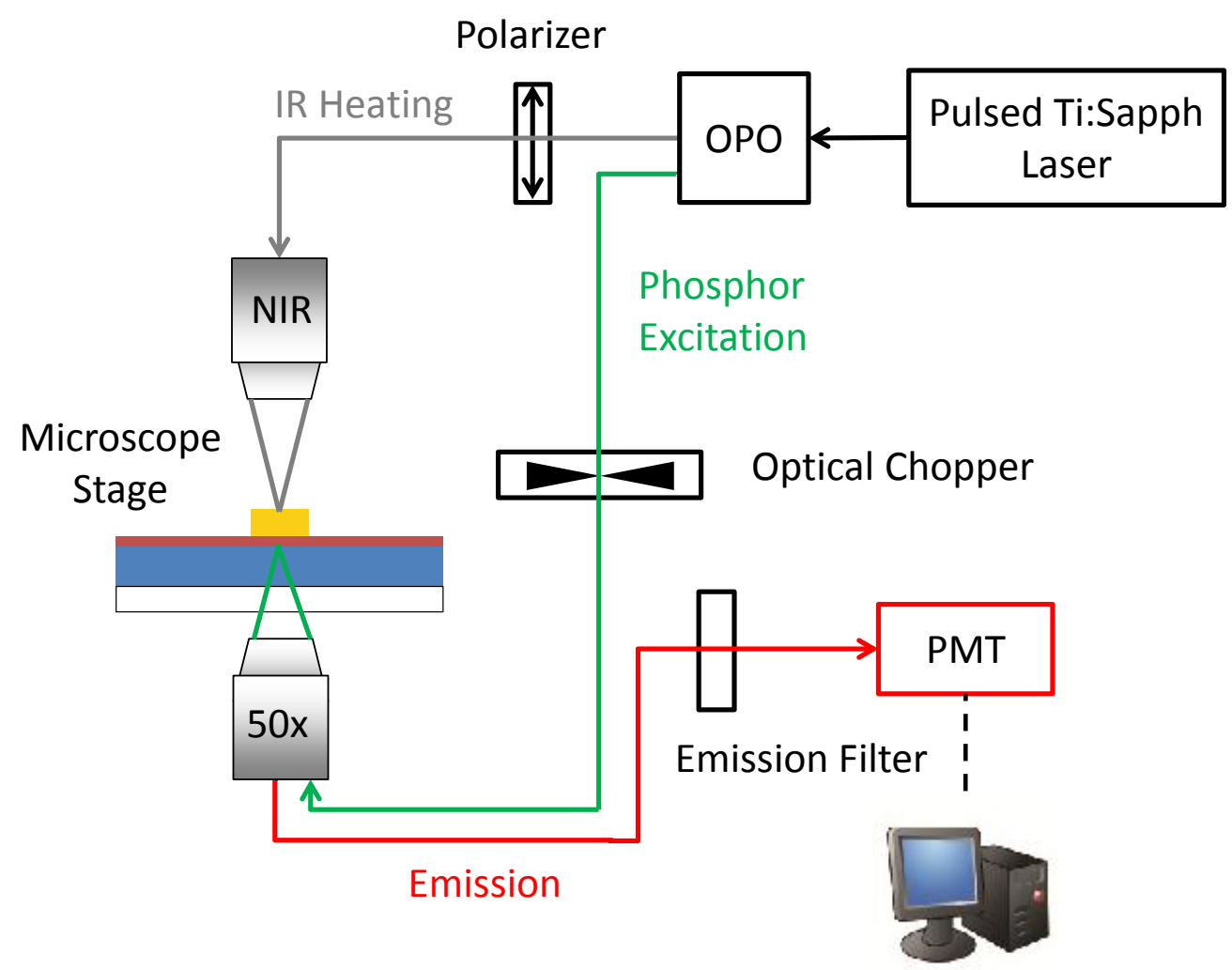

Figure 23. Thin-film TGP thermal microscopy schematic. 


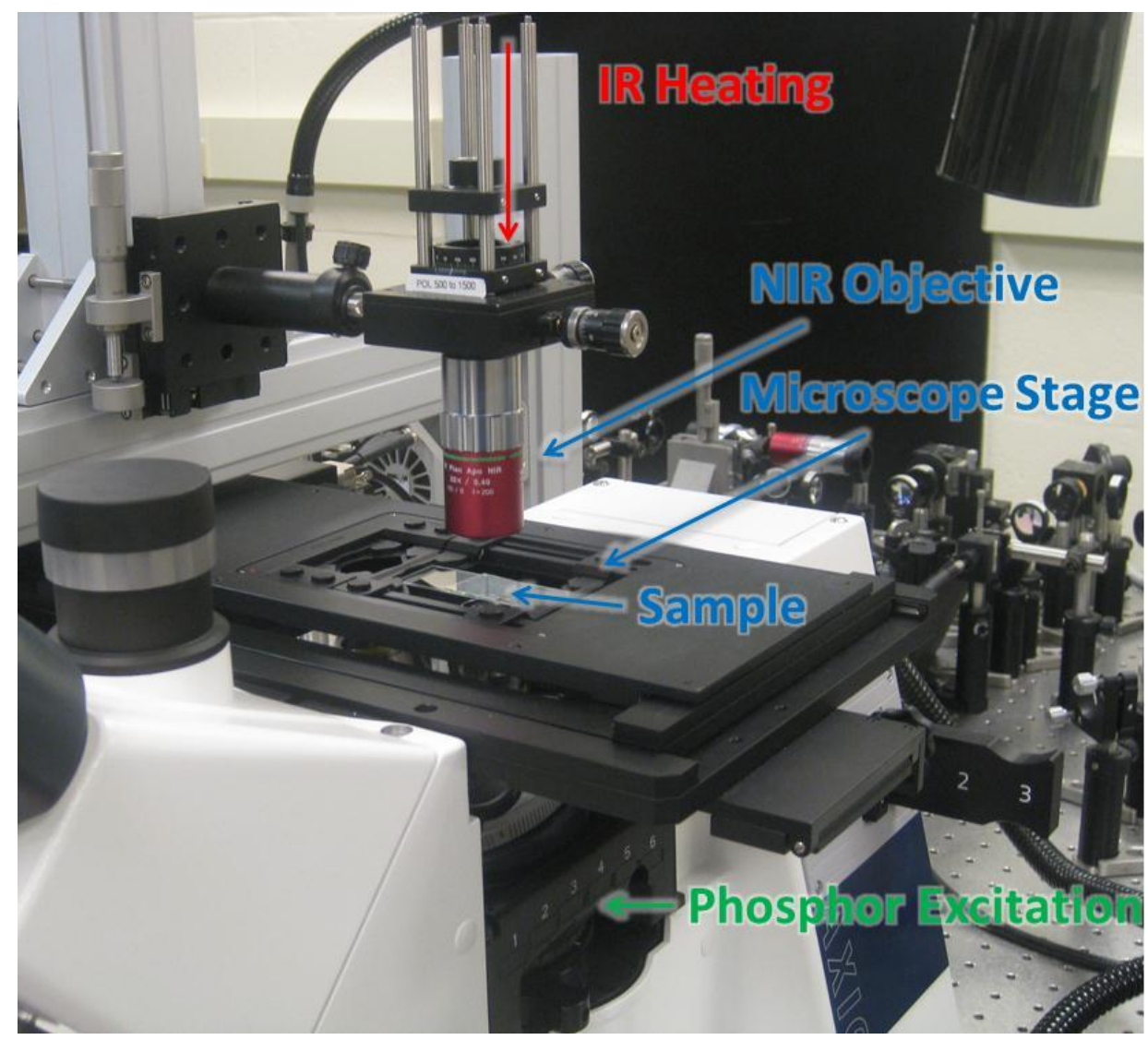

Figure 24. Picture of thermal microscopy setup.

The emission of the phosphor was collected though the bottom objective lens and sent out of the microscope into a $694 \mathrm{~nm}$ bandpass filter, Figure 25. This filter was used to transmit only the temperature dependent emission wavelength of the phosphor ${ }^{40}$. Past the filter, the emission was routed to a fiber-coupled photomultiplier tube (PMT) to detect the phosphor decay. The PMT uses a photoelectric material to convert photons into an electrical signal. The electrical signal was recorded by a digitizer that was triggered to read during the off state of the phosphor excitation. Over 400 decay readings were recorded and averaged for each measurement to increase the signal-to-noise ratio. 


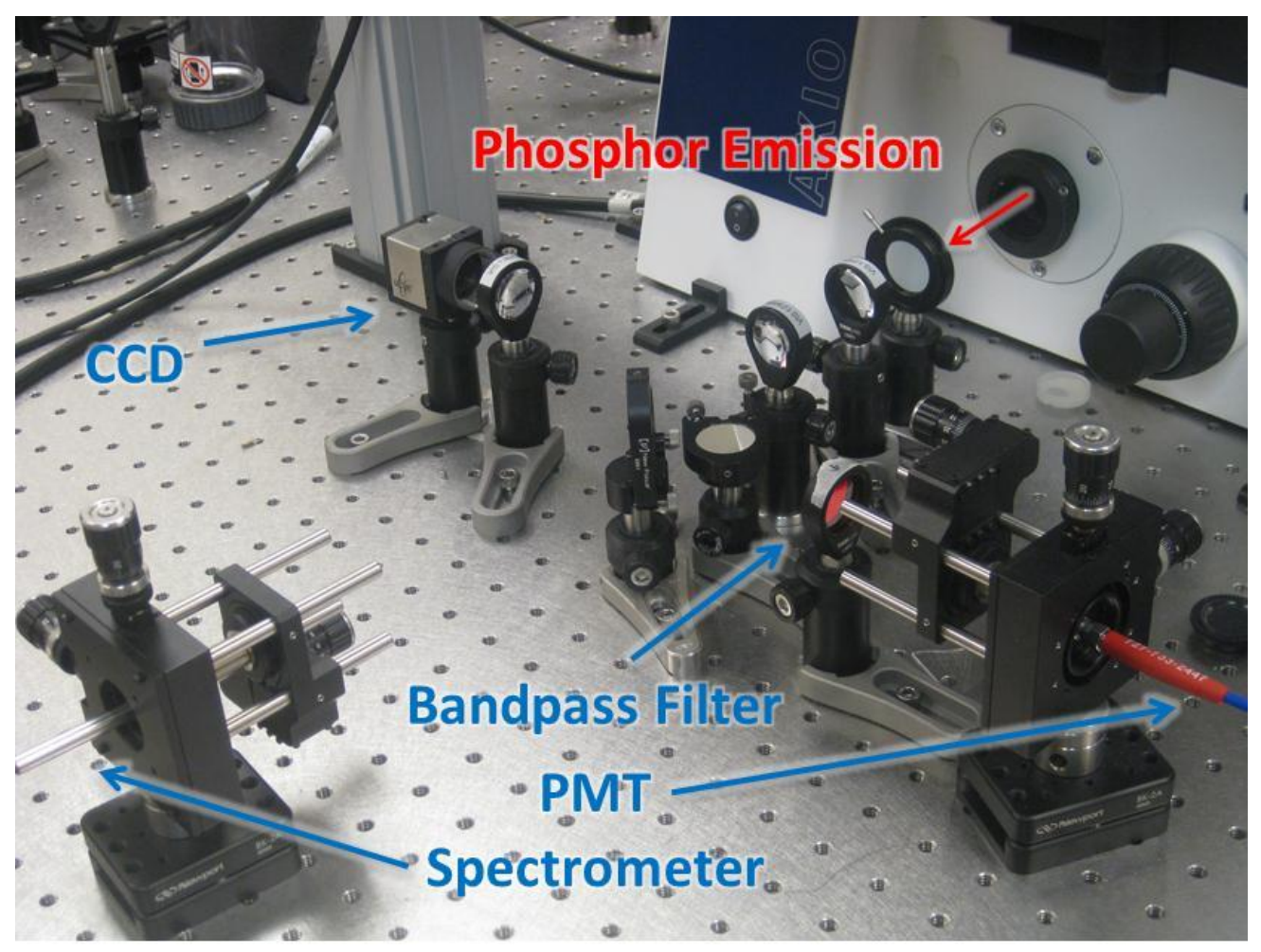

Figure 25. Picture of microscope export. Mirrors allowed for signal to be sent to CCD, spectrometer, or PMT.

The data of the averaged decay readings for this particular ruby phosphor exhibited a double exponential decay. The log plot in Figure 26 shows that the phosphor yields a faster initial decay followed by a slower second decay. For the measurements in this thesis, the second decay was the only decay time used to calculate temperatures. This is because the second decay gave more consistent results and its values more closely matched other ruby decay time measurements in literature ${ }^{38-40}$. At the time of this writing, it is not yet understood what mechanism causes the initial, faster decay. 


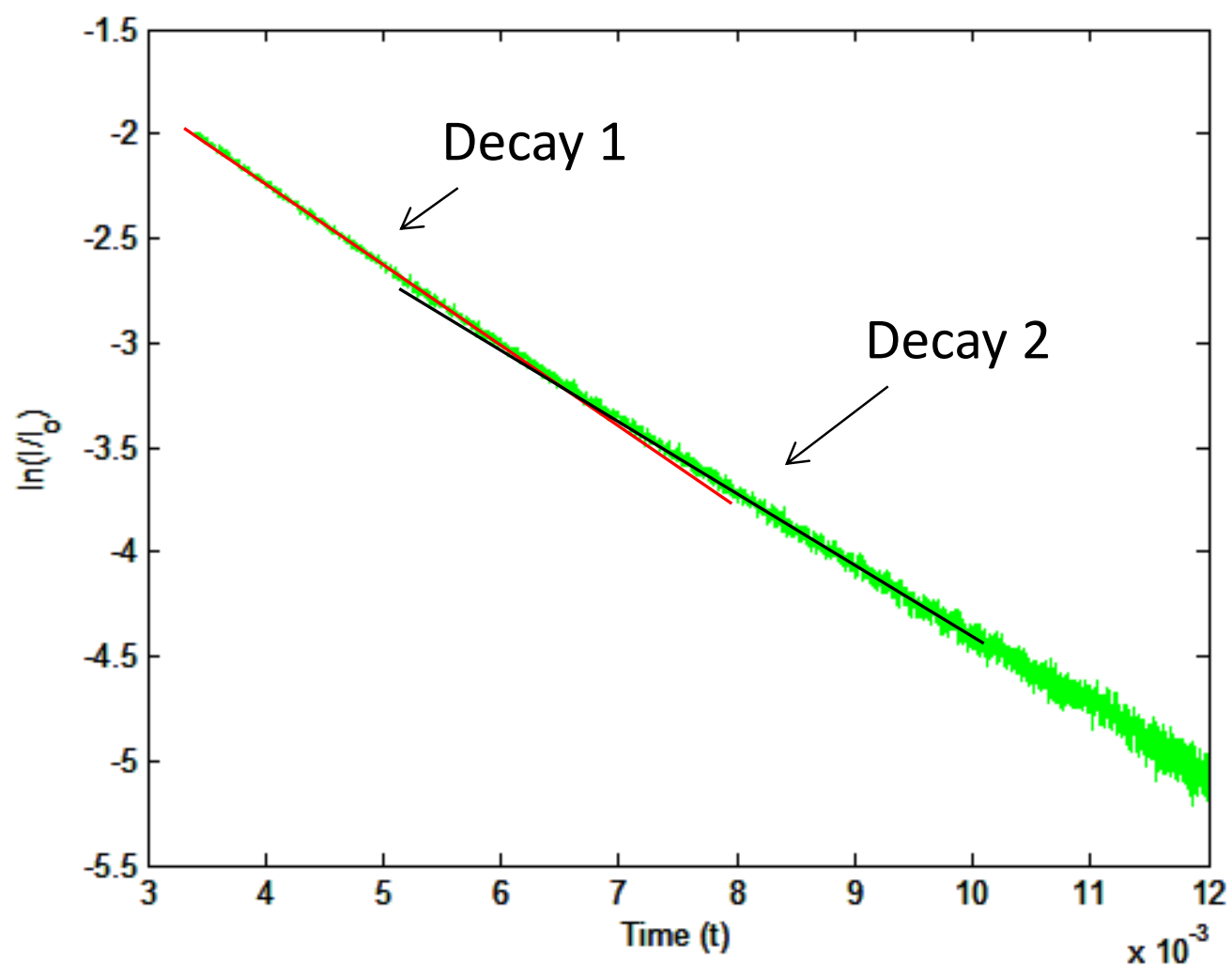

Figure 26. Log plot of an averaged decay reading from the ruby phosphor. The slope of the exponential function in log space indicates phosphor decay time

One issue with this measurement technique is that the metal in the antenna structures alters the phosphor film's decay time. This is a well-known phenomenon and occurs because metal objects near an emitter offer additional non-radiative de-excitation pathways for the excited electrons ${ }^{42}$. As a result, the decay time decreases giving a false temperature reading. Table 1 shows a table of measurements taken without any external heating on the diabolo array, the dipole array, and off the arrays. To account for this decay-altering effect, calibration data of the phosphor was performed with the excitation beam focused onto the diabolo antenna array. This way any effects caused by the metal were incorporated into the measured lifetimes. 


\section{Table 1}

Effects of Metal on TGP Decay Time Measurements

\begin{tabular}{cc}
\hline Measurement Location & Decay Time (ms) \\
\hline Bare ruby film & 3.080 \\
Diabolo array & 2.891 \\
Dipole array & 2.898 \\
\hline
\end{tabular}

\section{Calibration and Array Temperature Measurements}

Calibration of the ruby phosphor was necessary to match decay times with absolute local temperatures. To replicate the conditions of the actual measurement, the calibration was performed in the microscopy setup of Figure 23. The sample was placed on an ITO coated glass slide, which acted as a transparent resistive heating element. A voltage applied to the ITO layer uniformly heated the sample and a thermocouple placed on top of the ruby layer measured the phosphor temperature. Figure 27 shows the schematic of the calibration setup. 


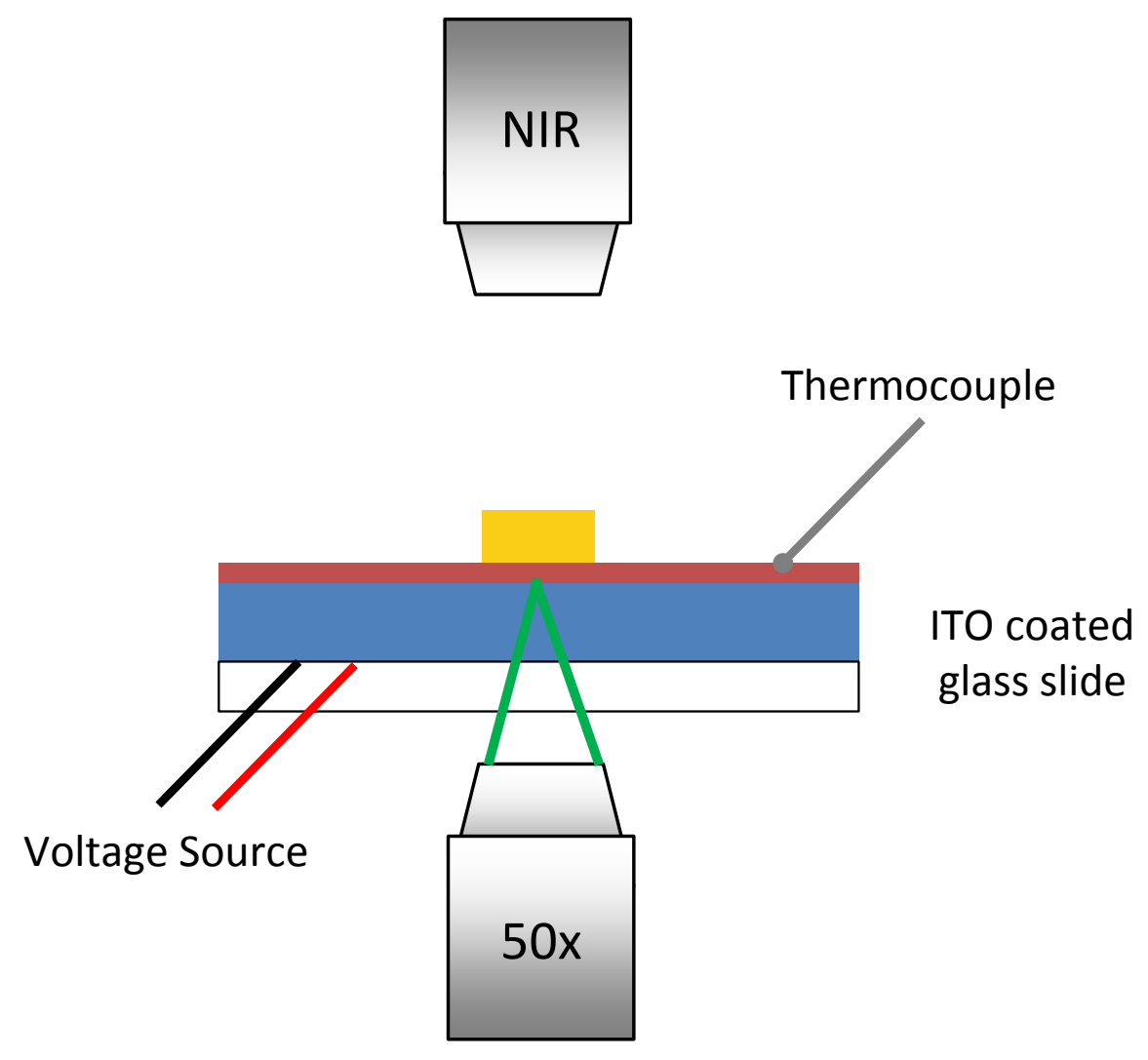

Figure 27. Schematic of calibration setup.

The voltage applied the ITO layer was varied to change the temperature of the sample, and at steady-state conditions the phosphor decay time was measured. Steadystate was confirmed by taking several measurements until the decay time stabilized. Figure 28 shows the results from the calibration. At each temperature, 10 decay measurements were taken. The error bars in Figure 28 represent the $95 \%$ confidence interval of the data. 


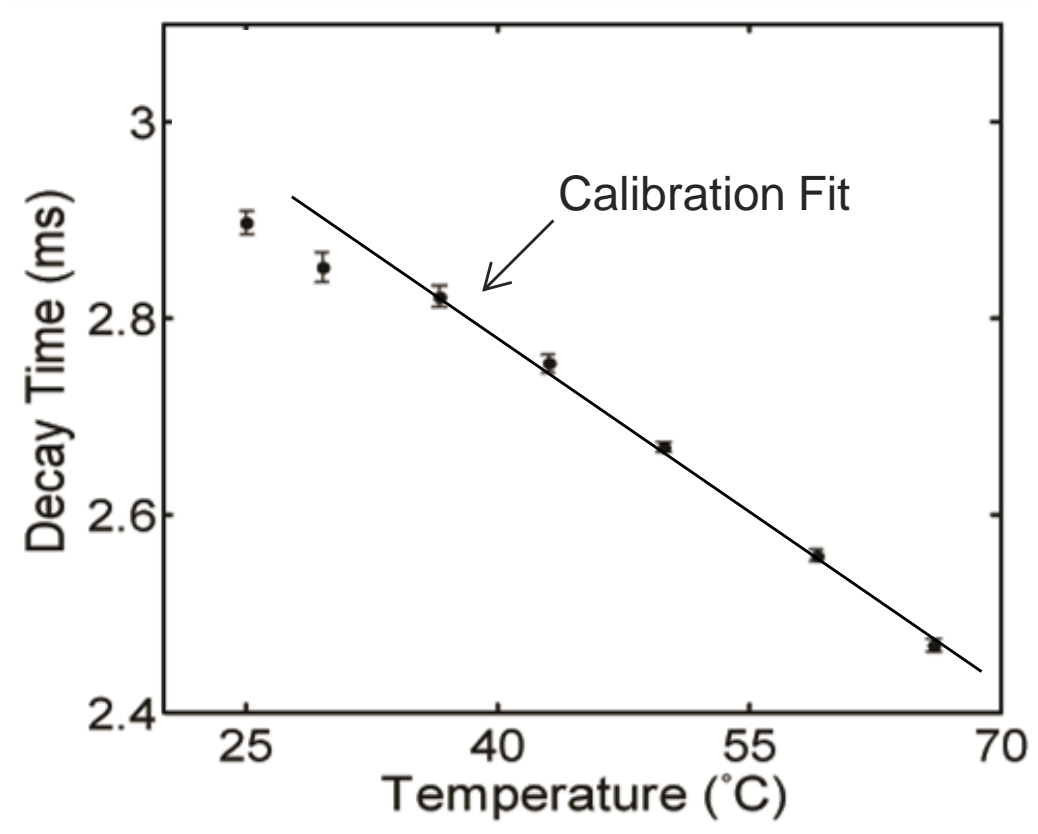

Figure 28. Calibration curve of $100 \mathrm{~nm}$ ruby TGP.

After calibrating the phosphor and allowing the sample to cool, the antenna arrays were optically heated and their temperatures were measured. For the measurements, the IR heating beam was polarized along the optical axis of the arrays and the power was set to $125 \mathrm{~mW}$. The beam was focused onto each array with a measured full-width-at-halfmaximum of $19.5 \mu \mathrm{m}$ resulting in an input intensity of $0.42 \mathrm{~mW} / \mu \mathrm{m}^{2}$. After reaching steady-state conditions, decay times were recorded on each array and fit to the calibration curve. The resulting temperatures are displayed in Figure 29. 


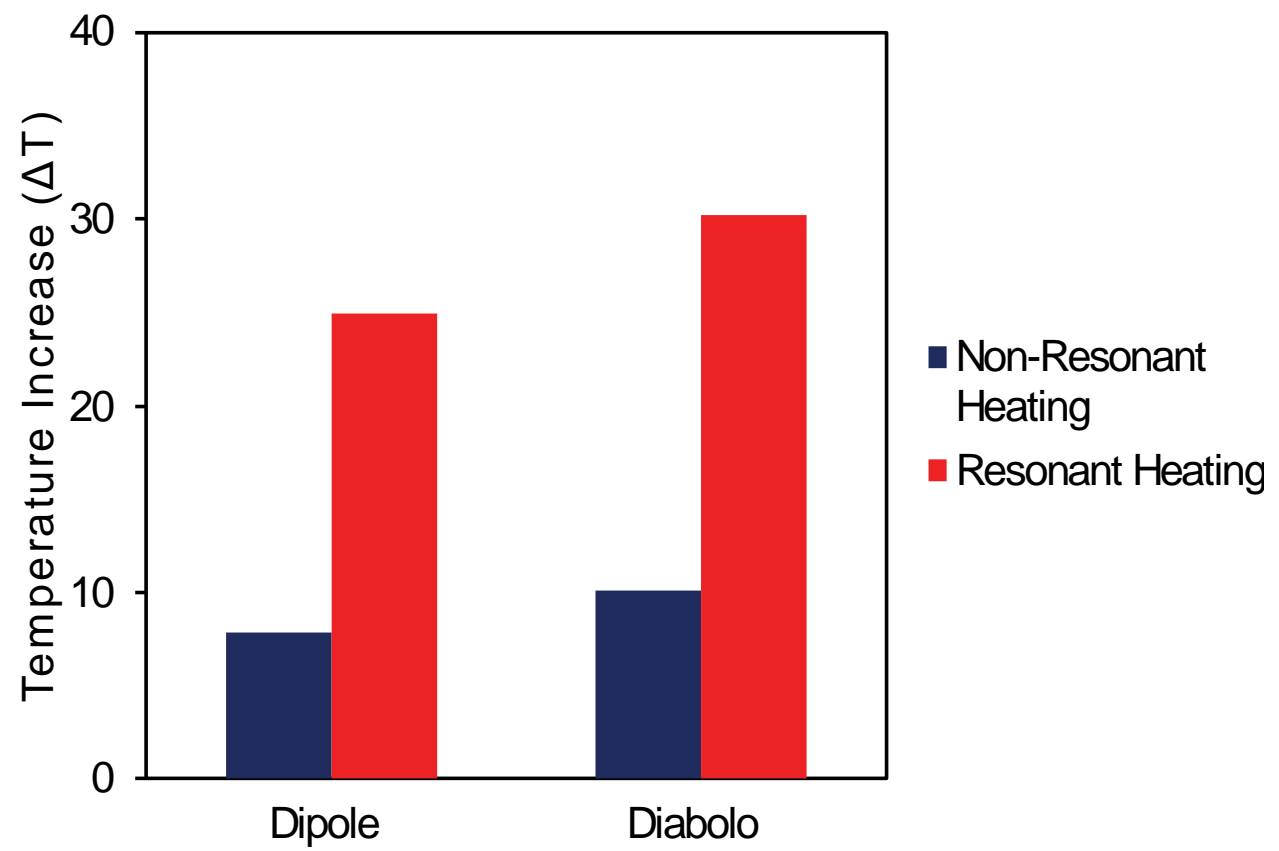

Figure 29. Temperature measurements of optically excited dipole and diabolo arrays with non-resonant (blue) and resonant (red) polarizations. $\Delta \mathrm{T}$ is temperature rise over the ambient temperature.

The diabolo and dipole antenna arrays show temperature rises $(\Delta \mathrm{T})$ of $30.2^{\circ} \mathrm{C}$ and $24.9^{\circ} \mathrm{C}$ respectively. These results validate the proposed thermoplasmonic design rationale by showing that the diabolo antenna, with its enhanced magnetic field, generates more heat than the dipole antenna. To verify that the heating occurred through the resonant excitation of surface plasmons, the heating beam was rotated $90^{\circ}$ to the off polarization where neither array has a strong resonance. As expected, the temperatures are only slightly increased due to non-resonant absorption in the metal nanostructures. 


\section{CHAPTER V}

\section{THERMAL MODELING}

The goal of this chapter is to present the two thermal modeling techniques used in this thesis. The first technique employs an analytical 3-D conduction model to calculate the temperatures of the optically heated nanoantenna arrays. The other method uses Green's function solutions to develop a spatial map of the temperature profile around the superstructure-enhanced diabolo nanoantenna. The assumptions and motivations for each model are discussed in detail and the calculated results are reported.

\section{Optically Heated Nanoantenna Arrays}

To confirm the experimental temperature measurements of the optically heated nanoantenna arrays, an analytical 3-D conduction model of the system was used in which the $\Delta T$ of the arrays could be found from the following equation,

$$
q=S k \Delta T
$$

where $\mathrm{S}$ is the conduction shape factor, $\mathrm{k}$ is the thermal conductivity of the substrate, $\Delta \mathrm{T}$ is the temperature rise over ambient temperature, and $\mathrm{q}$ is the heat generated by the $\operatorname{array}^{43}$. This model, as depicted in Figure 30, corresponds to a heated disk on a semiinfinite slab in which the top surface is assumed adiabatic. This is a good approximation of the experimental conditions for several reasons. 


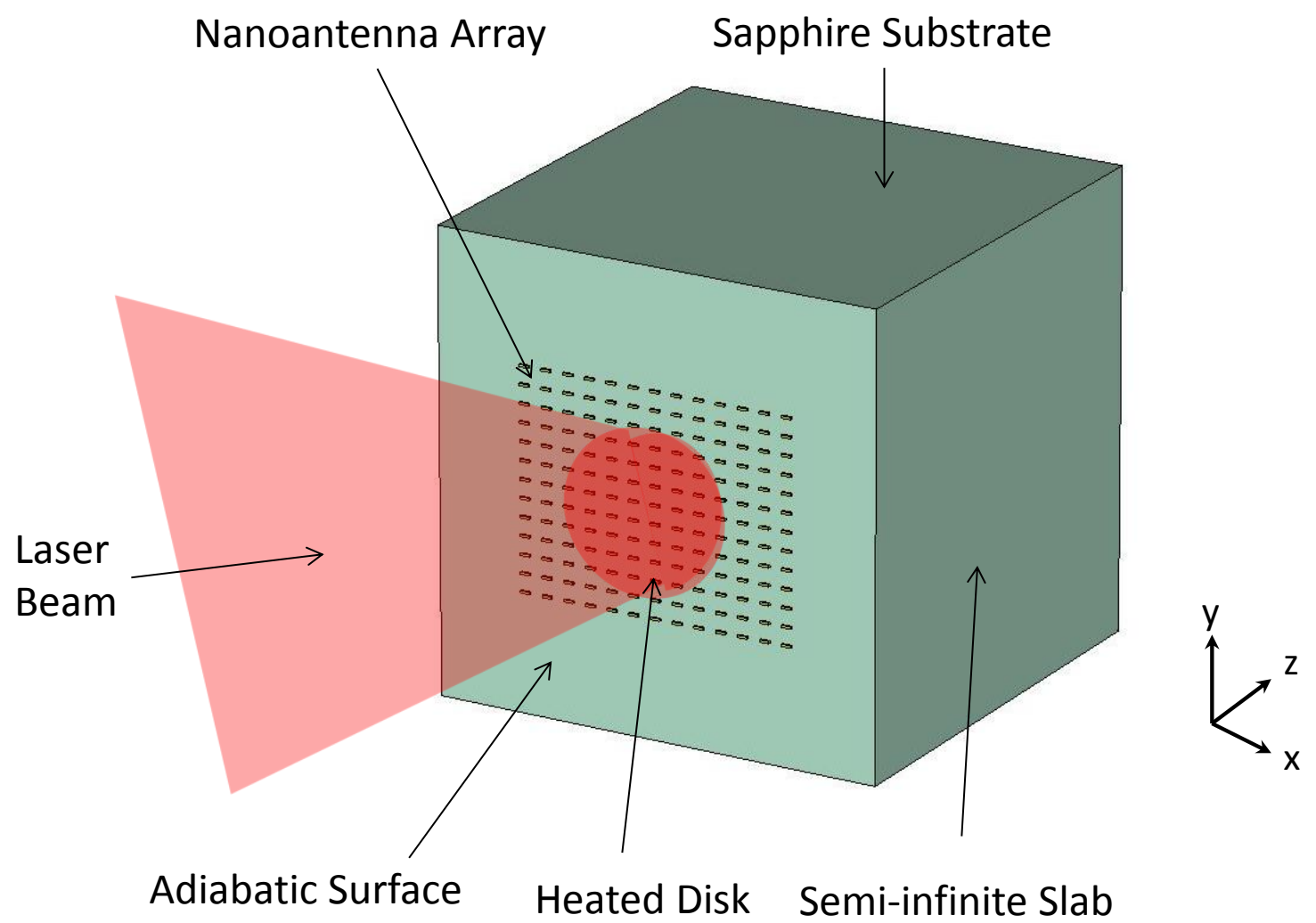

Figure 30. Depiction of the conduction model for a heated dipole antenna array.

First, the top surface can be assumed adiabatic due to the fact that the thermal conductivity of the sapphire substrate $(25 \mathrm{~W} / \mathrm{mK})$ is almost three orders of magnitude larger than the surrounding air medium $(0.026 \mathrm{~W} / \mathrm{mK})$. This disparity in thermal conductivity causes nearly all of the heat to flow out through the substrate and almost zero heat to flow through the air/sapphire interface. The heated disk assumption is also a good approximation of the experimental conditions because the heat generated in the arrays takes the shape of the focused infrared beam. Because the beam is round, the heat generation on the surface is round, or disk-like. 
For the assumption of semi-infinite medium, the half space of sapphire in Figure 30 should extend to infinity. In reality, this is a good assumption for the sapphire substrate's $x$ - and $y$-direction as they are on the order of centimeters and the beam size is on the order of microns. However, this is not an obvious assumption for the substrate's $z$ direction, which is only $430 \mu \mathrm{m}$. To check the validity of this assumption, the temperature rise over ambient was found at the bottom interface of the sapphire substrate assuming a half-space radial distribution of temperature from a heated sphere at the top interface,

$$
\Delta T=\frac{q}{2 \pi k r}
$$

where $r$ is $430 \mu \mathrm{m}$. Using the calculated heat generation of the array (details of this are shown later), the temperature rise at the bottom interface is found to be $0.45^{\circ} \mathrm{C}$. Because this value is an order of magnitude lower than the expected temperature rise in the arrays, the semi-infinite slab assumption is valid.

To calculate the $\Delta \mathrm{T}$ of the optically heated arrays from eq 15 , the shape conduction factor $(S)$ and the heat generation $(q)$ need to be determined. For a uniformly heated disk on top of a semi-infinite medium, which is the condition with laser illumination, the shape conduction factor becomes $\mathrm{S}=3 \pi^{2} \mathrm{D} / 16$, where $D$ is the laser beam diameter. In this case, the $\Delta T$ calculated in eq 15 corresponds to the average temperature rise of the heated disk over ambient temperature. This matches well with the conditions of the experimental temperature measurements as the TGP microscopy method measures an average temperature rise in the arrays over the entire beam area. 
The heat generation in the arrays was determined from simulated FDTD results of the diabolo and dipole antenna arrays. Using the reflection $(R)$ and transmission $(T)$ data, the absorption $(A)$ of each array could be found from $\mathrm{A}=1-\mathrm{R}-\mathrm{T}$. The heat generation was then calculated by multiplying the input power from the experiment $(125 \mathrm{~mW})$ by the absorption of each array. The diabolo array generated $30.3 \mathrm{~mW}$ of heat and the dipole array generated $22.5 \mathrm{~mW}$ of heat. The calculated results from the analytical model are shown in Figure 31 and are in good agreement with the experimentally measured temperatures.

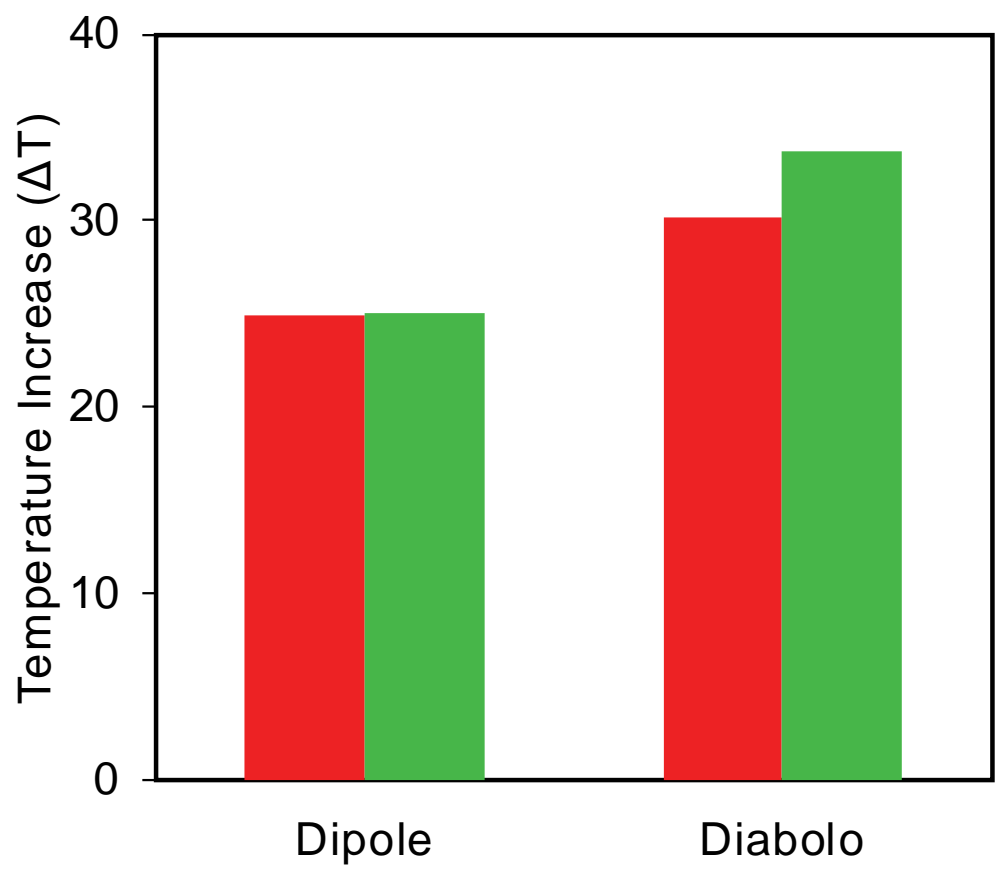

- Experimental Heating - Analytical Model

Figure 31. Experimental temperature measurements (red) and results from the analytical conduction model (green). 


\section{Nanoparticle Superstructure}

The nanoparticle superstructure geometry, which focuses grating-coupled electromagnetic energy onto a single diabolo antenna, provides both a highly confined and greatly intensified thermal hotspot. To show both of these attributes, a Green's function thermal model that calculates the magnitude and distribution of temperature around the diabolo antenna was used. Green's functions are built around the concept of summing the contributions of instantaneous, volumetric heat sources to gain insight into the total response of the system. This technique can be used in thermal modeling to develop conduction solutions, but it is also a popular method in other areas of science.

To build a conduction solution for the nanoparticle superstructure, volumetric heat sources (spheres) were arranged on the air/sapphire interface in the shape of a diabolo antenna, as shown in Figure 32. The heat generated in the diabolo antenna was divided equally among all the heat sources. Using a superposition of the Green's function, the temperature contributions of each spherical heat source at every point in the simulation

domain were summed together in order to get the total response of the diabolo structure ${ }^{27}$. Like the analytical model presented above, the air/sapphire interface was assumed adiabatic, and therefore the temperature distribution was only found in the sapphire substrate. To find the temperature at each point in the sapphire, the following equations were used:

$$
T(r)=\sum_{j=1}^{N} G\left(r, r_{i}\right) q
$$

and 


$$
G\left(r, r_{i}\right)=\frac{1}{2 \pi k R}
$$

where $\mathrm{T}(\mathrm{r})$ is the temperature rise over ambient temperature at a particular point in space, $G\left(r, r_{i}\right)$ is a scalar Green's function, $\mathrm{q}$ is the heat generated in a single spherical heat source, $\mathrm{k}$ is the thermal conductivity of the sapphire substrate, and $\mathrm{R}$ is the radial distance between a heat source and a point in the simulation domain. Because the heat sources were located above the air/sapphire interface, $R=\sqrt{\left(x-x^{\prime}\right)^{2}+\left(y-y^{\prime}\right)^{2}+(z-d)^{2}}$, where the primed coordinates represent the heat sources.

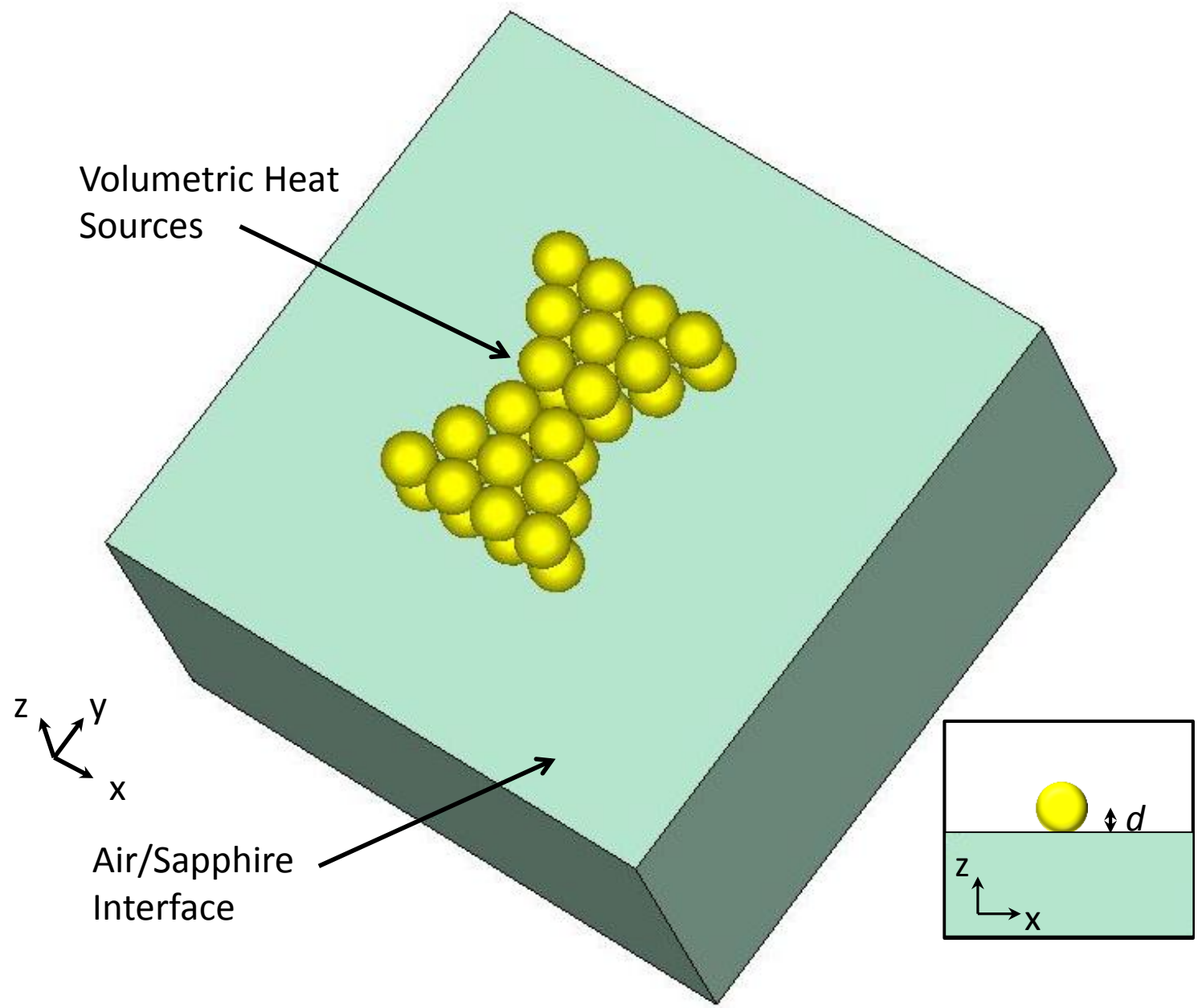

Figure 32. Depiction of the conduction model for the diabolo antenna. 
The Green's function shown in eq 18 was developed for this specific model by first applying the appropriate form of Fourier's law for radial heat conduction,

$$
q=-k A \frac{d T}{d r}
$$

where, $\mathrm{A}$ is the surface area of the sphere $\left(4 \pi \mathrm{r}^{2}\right)$ normal to the direction of heat flow ${ }^{43}$.

Then, assuming $\mathrm{q}$ and $\mathrm{k}$ are independent of $\mathrm{r}$, eq 19 takes the form,

$$
\begin{gathered}
\frac{q}{k 4 \pi} \int_{r_{o}}^{r} \frac{1}{r^{2}} d r=-\int_{T_{o}}^{T} d T \\
\Delta T(r)=\frac{q}{k 4 \pi R}
\end{gathered}
$$

In the case of an adiabatic top surface, heat dissipation is restricted to half of the spherical heat source and therefore eq 21 is reduced to,

$$
\Delta T(r)=\frac{q}{k 2 \pi R}=G\left(r, r_{i}\right) q
$$

To determine the heat generation input to eq 22, FDTD simulations were used to calculate the absorption loss in the diabolo antenna as both a single structure and a superstructure. Both a single diabolo and grating-enhanced diabolo were simulated in order to gain an understanding of the enhancement achieved by the superstructure. The input intensity for both simulations was set to $9 \mathrm{~mW} / \mu^{2}$. The results of the FDTD simulations and thermal modeling show that the superstructure greatly enhances the heat generation in the diabolo antenna as the superstructure produces a temperature rise of $327^{\circ} \mathrm{C}$ while the single diabolo antenna only produces a temperature rise of $45^{\circ} \mathrm{C}$. This large discrepancy is due to the increased energy coupling to the diabolo and the non- 
linear heat generation relationship of eq 6. The temperature maps presented in Figure $33 \mathrm{~b}, \mathrm{c}$ show that the diabolo antenna in the superstructure still retains a highly localized thermal hotspot, a property that is greatly desired in nanoscale science.
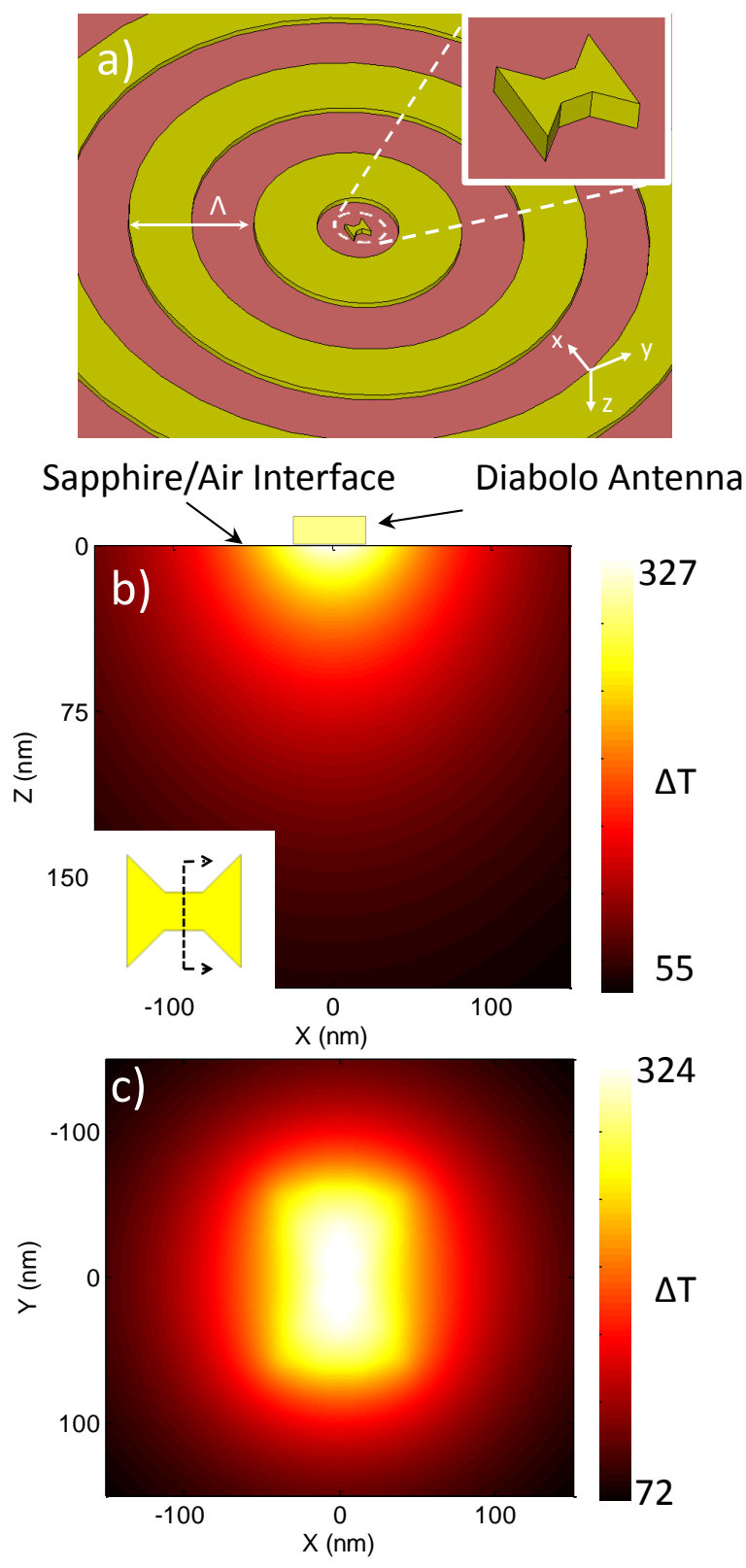

Figure 33. (a) Plasmonic superstructure made up of a diabolo antenna surrounded by 3 circular gratings: $\Lambda=956 \mathrm{~nm}$. Diabolo antenna dimensions are the same as Figure 2a. (b) Temperature profile below the grating enhanced diabolo antenna in the z-direction. (c) Temperature profile of grating enhanced diabolo antenna $3 \mathrm{~nm}$ below the surface in the $\mathrm{x}$ and $\mathrm{y}$ - direction. 


\section{CHAPTER VI}

\section{DISCUSSION}

In this discussion section, certain aspects of the proposed temperature measurement technique and design rationale are analyzed. For the thermal microscopy technique, a discussion is presented on the advantages and limitations of the method. Also, possible approaches to overcoming the limitations are suggested. For the design rationale, techniques for even further optimization of the designs presented in the previous sections of this thesis are discussed. This includes both the diabolo antenna and the superstructure

\section{TGP Thermal Microscopy Technique}

The proposed TGP thermal microscopy technique has several aspects that make it attractive for robust micro/nanoscale temperature measurements. First, TGP temperatures are correlated with decay times, which protects the measurement against excitation intensity fluctuations, concentration variation, and blinking ${ }^{40}$. Also, due to the fact that the TGP is in a solid state and has a high melting temperature, large temperature ranges can be probed. In fact, a spectrum of various phosphors shows temperature sensitivity from near $0 \mathrm{~K}$ to $1800 \mathrm{~K}^{37}$. 
This method, however, does have its drawbacks. The experimentally measured temperatures are lower than the actual thermoplasmonic structure temperature because the TGP thermal microscopy technique measures an average temperature throughout the $100 \mathrm{~nm}$ phosphor layer. The average temperature of the layer may not be an accurate representation of the thermoplasmonic structure, such as the case of the simulation in Figure $33 \mathrm{~b}$ which shows a large temperature gradient over a distance of $100 \mathrm{~nm}$. This problem, however, could be overcome with the use of 2-photon phosphor excitation (Figure 34). With this method, the phosphor is only excited at the focal point of the excitation source ${ }^{44}$. This allows the phosphor excitation to occur arbitrarily close to the thermoplasmonic structure, which will increase the accuracy of the measurement. Also, this technique opens the possibility of three-dimensional probing of temperatures around thermoplasmonic structures.

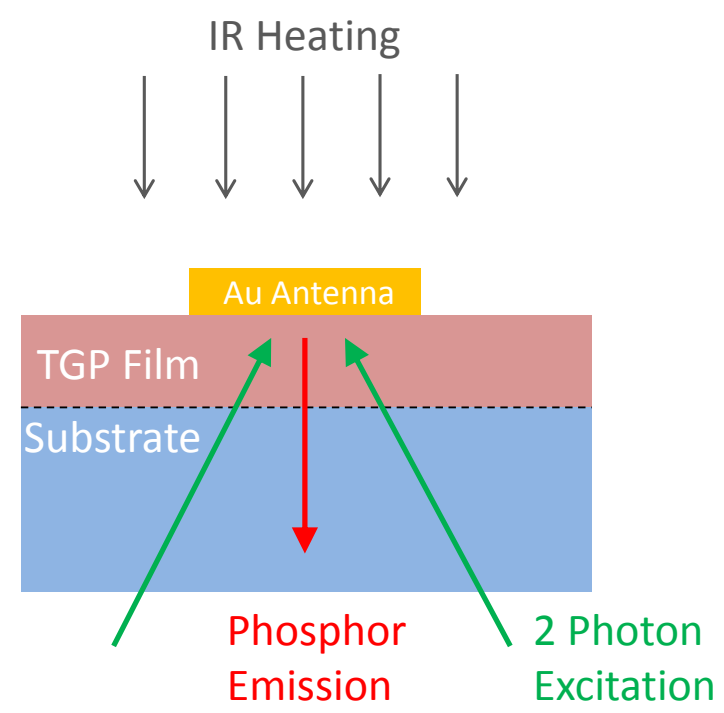

Figure 34. Two photon excitation of ruby phosphor layer. 
Another drawback to this technique is that the spatial resolution is limited by the diffraction limit of the excitation beam, which can be approximated as $d=\frac{\lambda}{2}$, where $\mathrm{d}$ is the smallest possible excitation beam spot size and $\lambda$ is the excitation beam wavelength. According to the diffraction limit relationship, the previous experimental measurement excitation source, $532 \mathrm{~nm}$, can only achieve a maximum possible resolution of $266 \mathrm{~nm}$. This makes it difficult to map temperatures around small thermoplasmonic designs, such as the diabolo antenna presented early. However, this problem could be overcome by using phosphors with different excitation wavelengths. Literature has reported several TGPs with deep-UV excitation wavelengths, thus increasing the maximum resolution to sub-150 $\mathrm{nm}^{37}$.

\section{Design Rationale}

The diabolo antenna fabricated in this thesis could be further optimized to enhance its thermoplasmonic effect. Other studies of the diabolo antenna have shown $\mathrm{H}$ field enhancements of almost 600 times at wavelengths near 2.5 microns ${ }^{29}$. These larger enhancements occur due to a smaller neck size $(20 \mathrm{~nm})$ and larger arm length $(145 \mathrm{~nm})$ of the antenna. This increased ratio between the arm length and neck size promotes more charge funneling through the neck region and thus enhances the magnetic field around the structure. Feature sizes of these dimensions could be realized with high-end EBL tools.

The superstructure takes advantage of the diabolo antenna's enhanced thermoplasmonic design by incorporating the antenna with a plasmonic lens to couple in 
more electromagnetic energy. In the thermal modeling chapter, the super structure achieved a $327^{\circ} \mathrm{C}$ temperature increase. Though this is a large value, the design could be even further enhanced to promote an even larger temperature rise. By adding more circular gratings to the lens and using a lower thermal conductivity substrate, the diabolo temperature will increase $\mathrm{e}^{45}$. This is because additional rings, up to a certain point, couple in more electromagnetic energy and lower conductivity substrates act as thermal insulators. Making these two improvements will lower the power requirements of the heating beam and make the superstructure a more attractive candidate for heat assisted magnetic recording. Also, fabrication of these enhanced devices should be relatively simple using the techniques described in Chapter 3. 


\section{CHAPTER VII}

\section{CONCLUSION}

This thesis proposes two promising tools for the field of thermoplasmonics: a general design rationale for nanoscale heat sources and a technique for robust thermal microscopy. The simulations and experiments throughout this work have proven that enhanced magnetic fields and current densities in thermoplasmonic structures will increase their heat generation and temperature. Moreover, it has been shown that dramatic increases in heat generation are possible with a superstructure design due to increased energy coupling and the non-linear scaling relationship of eq 6. Also, the TGP thermal microscopy technique is proven throughout this work to be a robust temperature

measurement technique for thermoplasmonic structures. The versatility and advantages of the technique make it a strong candidate for widespread adoption in micro/nanoscale science. These two new tools presented in this thesis will hopefully fuel the continued emergence of the thermoplasmonics field and help facilitate the integration of thermoplasmonics into critical applications. 


\section{REFERENCES}

1. Schuller, J. A. et al. Plasmonics for extreme light concentration and manipulation. Nature materials 9, 193-204 (2010).

2. Govorov, A. O. \& Richardson, H. H. Generating heat with metal nanoparticles. Nano Today 2, 30-38 (2007).

3. Baffou, G., Quidant, R. \& Girard, C. Heat generation in plasmonic nanostructures: Influence of morphology. Applied Physics Letters 94, 153109 (2009).

4. Baffou, G., Girard, C. \& Quidant, R. Mapping Heat Origin in Plasmonic Structures. Physical Review Letters 104, 136805 (2010).

5. Baffou, G., Quidant, R. \& de Abajo, F. J. G. Nanoscale Control of Optical Heating in Complex Plasmonic Systems. ACS nano 4, 709-716 (2010).

6. Han, G., Ghosh, P., De, M. \& Rotello, V. M. Drug and gene delivery using gold nanoparticles. NanoBiotechnology 3, 40-45 (2007).

7. Huschka, R. et al. Light-induced release of DNA from gold nanoparticles: nanoshells and nanorods. Journal of the American Chemical Society 133, 1224755 (2011).

8. Loo, C., Lowery, A., Halas, N., West, J. \& Drezek, R. Immunotargeted nanoshells for integrated cancer imaging and therapy. Nano letters 5, 709-11 (2005).

9. Jain, P. K., El-Sayed, I. H. \& El-Sayed, M. A. Au nanoparticles target cancer. Nano Today 2, 18-29 (2007).

10. Challener, W. A. et al. Heat-assisted magnetic recording by a near-field transducer with efficient optical energy transfer. Nature Photonics 3, 220-224 (2009).

11. Stipe, B. C. et al. Magnetic recording at $1.5 \mathrm{~Pb} \mathrm{~m}-2$ using an integrated plasmonic antenna. Nature Photonics 4, 484-488 (2010).

12. Adleman, J. R., Boyd, D. A., Goodwin, D. G. \& Psaltis, D. Heterogenous Catalysis Mediated by Plasmon Heating. Nano letters 9, 4417-4423 (2009).

13. Cao, L., Barsic, D. N., Guichard, A. R. \& Brongersma, M. L. Plasmon-assisted local temperature control to pattern individual semiconductor nanowires and carbon nanotubes. Nano letters 7, 3523-7 (2007). 
14. Christopher, P., Xin, H. \& Linic, S. Visible-light-enhanced catalytic oxidation reactions on plasmonic silver nanostructures. Nature chemistry 3, 467-72 (2011).

15. Linic, S., Christopher, P. \& Ingram, D. B. Plasmonic-metal nanostructures for efficient conversion of solar to chemical energy. Nature materials 10, 911-21 (2011).

16. Wang, L. \& Li, B. Thermal Memory: A Storage of Phononic Information. Physical Review Letters 101, 267203- (2008).

17. Yang, N., Zhang, G. \& Li, B. Thermal Rectification In Asymmetric Graphene Ribbons. (2009).at <http://arxiv.org/abs/0906.1046>

18. Kim, W. J. et al. Photothermal-reaction-assisted two-photon lithography of silver nanocrystals capped with thermally cleavable ligands. Applied Physics Letters $\mathbf{9 8}$, 133110 (2011).

19. Aydin, K., Ferry, V. E., Briggs, R. M. \& Atwater, H. A. Broadband polarizationindependent resonant light absorption using ultrathin plasmonic super absorbers. Nature communications 2, 517 (2011).

20. Liu, X., Starr, T., Starr, A. F. \& Padilla, W. J. Infrared Spatial and Frequency Selective Metamaterial with Near-Unity Absorbance. Physical Review Letters 104, 207403- (2010).

21. Govorov, A. O. et al. Gold nanoparticle ensembles as heaters and actuators: melting and collective plasmon resonances. Nanoscale Research Letters 1, 84-90 (2006).

22. Yue, Y. \& Wang, X. Nanoscale thermal probing. Nano Reviews 3, (2012).

23. Maier, S. A. \& Atwater, H. A. Plasmonics: Localization and guiding of electromagnetic energy in metal/dielectric structures. Journal of Applied Physics 98, 011101 (2005).

24. Novotny, L. \& van Hulst, N. Antennas for light. Nature Photonics 5, 83-90 (2011).

25. Baffou, G. \& Rigneault, H. Femtosecond-pulsed optical heating of gold nanoparticles. Physical Review B 84, 035415- (2011).

26. Baffou, G. \& Quidant, R. Thermo-plasmonics: using metallic nanostructures as nano-sources of heat. Laser \& Photonics Reviews $\mathrm{n} / \mathrm{a}-\mathrm{n} / \mathrm{a}$ (2012).doi:10.1002/lpor.201200003 
27. Baffou, G., Quidant, R. \& Girard, C. Thermoplasmonics modeling: A Green's function approach. Physical Review B 82, 165424- (2010).

28. Sadiku, M. Elements of Electromagnetics. (Oxford University Press, USA: New York, 2010).

29. Grosjean, T., Mivelle, M., Baida, F. I., Burr, G. W. \& Fischer, U. C. Diabolo nanoantenna for enhancing and confining the magnetic optical field. Nano letters 11, 1009-13 (2011).

30. Zhou, N., Kinzel, E. C. \& Xu, X. Complementary bowtie aperture for localizing and enhancing optical magnetic field. Optics Letters 36, 2764 (2011).

31. Vial, A. \& Laroche, T. Description of dispersion properties of metals by means of the critical points model and application to the study of resonant structures using the FDTD method. Journal of Physics D: Applied Physics 40, 7152-7158 (2007).

32. Liu, Z. et al. Focusing surface plasmons with a plasmonic lens. Nano letters $\mathbf{5}$, 1726-9 (2005).

33. Majumdar, A. Scanning Thermal Microscopy. Annual Reviews 29, 505-585 (1999).

34. Cahill, D. G., Goodson, K. \& Majumdar, A. Thermometry and Thermal Transport in Micro/Nanoscale Solid-State Devices and Structures. Journal of Heat Transfer 124, 223 (2002).

35. Baffou, G., Kreuzer, M. P., Kulzer, F. \& Quidant, R. Temperature mapping near plasmonic nanostructures using fluorescence polarization anisotropy. Optics Express 17, 3291 (2009).

36. Carlson, M. T., Khan, A. \& Richardson, H. H. Local temperature determination of optically excited nanoparticles and nanodots. Nano letters 11, 1061-9 (2011).

37. Allison, S. W. \& Gillies, G. T. Remote thermometry with thermographic phosphors: Instrumentation and applications. Review of Scientific Instruments $\mathbf{6 8}$, 2615 (1997).

38. Pflitsch, C., Viefhaus, D. \& Atakan, B. CVD of Thin Ruby Films on Si(100) and Stainless Steel for Surface Temperature Sensor Applications. Chemical Vapor Deposition 13, 420-426 (2007).

39. Pflitsch, C., Siddiqui, R. A. \& Atakan, B. Phosphorescence properties of sol-gel derived ruby measured as functions of temperature and $\mathrm{Cr} 3+$ content. Applied Physics A 90, 527-532 (2007). 
40. Atakan, B., Eckert, C. \& Pflitsch, C. Light emitting diode excitation of Cr 3+ :Al 2 O 3 as thermographic phosphor: experiments and measurement strategy. Measurement Science and Technology 20, 075304 (2009).

41. Hu, W. (Walter), Sarveswaran, K., Lieberman, M. \& Bernstein, G. H. Sub-10 nm electron beam lithography using cold development of poly(methylmethacrylate). Journal of Vacuum Science \& Technology B: Microelectronics and Nanometer Structures 22, 1711 (2004).

42. Shimizu, K., Woo, W., Fisher, B., Eisler, H. \& Bawendi, M. Surface-Enhanced Emission from Single Semiconductor Nanocrystals. Physical Review Letters 89, 117401-(2002).

43. Incropera, F. P. \& DeWitt, D. P. Introduction To Heat Transfer. 912 (Wiley: Hoboken, 2007).

44. Oheim, M., Michael, D. J., Geisbauer, M., Madsen, D. \& Chow, R. H. Principles of two-photon excitation fluorescence microscopy and other nonlinear imaging approaches. Advanced drug delivery reviews 58, 788-808 (2006).

45. Steele, J. M., Liu, Z., Wang, Y. \& Zhang, X. Resonant and non-resonant generation and focusing of surface plasmons with circular gratings. Optics Express 14, 5664 (2006). 